\title{
AN EXISTENCE RESULT \\ FOR A NONCONVEX VARIATIONAL PROBLEM VIA REGULARITY
}

\author{
Irene Fonseca $^{1}$, Nicola Fusco ${ }^{2}$ And PaOlo Marcellini ${ }^{3}$
}

\begin{abstract}
Local Lipschitz continuity of minimizers of certain integrals of the Calculus of Variations is obtained when the integrands are convex with respect to the gradient variable, but are not necessarily uniformly convex. In turn, these regularity results entail existence of minimizers of variational problems with non-homogeneous integrands nonconvex with respect to the gradient variable. The $x$-dependence, explicitly appearing in the integrands, adds significant technical difficulties in the proof.
\end{abstract}

Mathematics Subject Classification. 49J45, 49K20, 35F30, 35R70.

Received May 9, 2001.

\section{INTRODUCTION}

In this paper we establish existence and regularity of minimizers of energy integrals of the type

$$
\int_{\Omega} f(x, D u(x)) \mathrm{d} x
$$

subject to Dirichlet boundary conditions. The main feature of our problem is the fact that the integrand $f=f(x, \xi)$ is not convex with respect to the gradient variable $\xi$.

In recent years the study of non convex variational problems has undergone remarkable developments, motivated in part by advances in the study of material stability and instability. Contemporary issues such as phase transitions in certain alloys (see [3,4]), nucleation [20], the onset of microstructure, and optimal design problems for thin films [18], require a good understanding of existence of (classical or generalized) equilibrium solutions for nonconvex energies. In addition, qualitative information on quasistatic solutions (e.g. regularity, hysteresis, oscillatory behavior) are needed in order to develop the evolutionary framework, and, in particular, to search for the dynamical evolution of phase boundaries. These issues have challenged traditional theories.

Keywords and phrases: Nonconvex variational problems, uniform convexity, regularity, implicit differential equations.

1 Carnegie-Mellon University, Pittsburgh, Pennsylvania 15213, U.S.A.; e-mail: fonseca@andrew.cmu.edu

2 Dipartimento di Matematica "R. Caccioppoli", Università di Napoli, Via Cintia, 80126 Napoli, Italy;

e-mail: fusco@matna1.dma.unina.it

3 Dipartimento di Matematica "U. Dini", Università di Firenze, Viale Morgagni 67 A, 50134 Firenze, Italy;

e-mail: marcell@udini.math.unifi.it 
Within this setting, a relevant example has been considered by Ball-James [3, 4], who studied the two "potential wells" problem:

$$
\operatorname{minimize} \int_{\Omega} f(D u(x)) \mathrm{d} x,
$$

where $u: \Omega \subset \mathbb{R}^{n} \rightarrow \mathbb{R}^{n}$ is a vector-valued function, $f: \mathbb{R}^{n \times n} \rightarrow[0,+\infty)$ is identically zero on two distinct potential wells $S O(n) \xi, S O(n) \eta$ and $f>0$ elsewhere. Here $\xi, \eta \in \mathbb{R}^{n \times n}$ and $S O(n)$ stands for the special orthogonal group. The existence of minimizers for the two potential wells problem has been obtained in two dimensions (i.e., $n=2$ ) by Dacorogna-Marcellini [10] and by Müller-Šverák [26] (for the case $n=3$ see also Dolzmann-Kirchheim-Müller-Šverák [15]). Nothing is known in higher dimension or for general integrands $f$ as in (1).

In this paper we restrict ourselves to the scalar-valued case, as a starting point to approach the vectorial setting. Also, the scalar-valued case is still far from being completely understood, unless the integrand $f$ depends only on the gradient variable $\xi$ and some special assumptions are made on the boundary data (see the references quoted below). Here we consider general boundary data $u_{0} \in W^{1, p}(\Omega), p>1$, and we allow the nonconvex integrands $f$ to explicitly depend on $x$ as in (1). In the proofs of the attainment results presented below the $x$-dependence introduces substantial technical difficulties.

The proof of the existence results for nonconvex variational problems considered in this paper hinges on the local Lipschitz continuity of minimizers of the relaxed problem associated to the bipolar $f^{* *}$ of $f$. These regularity results are presented in Section 2, and they apply to minimizers of some integrals of the Calculus of Variations with integrands $f^{* *}(x, \xi)$ convex with respect to $\xi \in \mathbb{R}^{n}$, but not everywhere uniformly convex; hence, we believe that the regularity results presented in Section 2 should be of interest by themselves.

In Section 3 we consider the variational problem

$$
\inf \left\{\int_{\Omega} f(x, D u(x)) \mathrm{d} x: u \in u_{0}+W_{0}^{1, p}(\Omega)\right\},
$$

where $u_{0} \in W^{1, p}(\Omega)$ is a given boundary datum and $f=f(x, \xi)$ is a continuous function satisfying some growth conditions similar to the ones considered in the previous Section 2, so as to ensure Lipschitz continuity of minimizers of the relaxed problem. Here the most relevant fact is that $f$ may be nonconvex with respect to the variable $\xi \in \mathbb{R}^{n}$.

It is known that the variational problem (2) may lack a minimizer (see Marcellini [22]; see also [6, 12, 21]). In the examples of nonexistence the following condition, expressed in terms of the bipolar $f^{* *}$ of $f$, is violated: for every $x \in \Omega$ the function $f^{* *}(x, \cdot)$ is affine on the set

$$
A(x)=\left\{\xi \in \mathbb{R}^{n}: f(x, \xi)>f^{* *}(x, \xi)\right\},
$$

i.e., there exist a continuous function $q$ and a vector field $m$ of class $C^{1}$, defined in the open set $\Omega_{A}:=$ $\{x \in \Omega: A(x) \neq \emptyset\}$, such that

$$
f^{* *}(x, \xi)=q(x)+\langle m(x), \xi\rangle, \quad \forall x \in \Omega_{A}, \quad \xi \in A(x)
$$

We also assume that the boundary (more precisely, the part of the boundary in $\Omega$ ) of the set

$$
\left\{x \in \Omega_{A}: \operatorname{div} m(x)=0\right\}
$$

has zero ( $n$-dimensional) measure.

In this paper we prove that $(3,4)$ (see also the more general assumptions made in Sect. 3.2) are sufficient conditions for existence of minimizers to the variational problem (2). We emphasize that we do not require any other condition on the vector field $m$ other than (4); in particular, we do not assume that the vector field $m$ has 
null divergence. We notice that, while condition (3) is necessary for guaranteeing the existence of minimizers (see $[6,21,22]$ ), we do not know whether condition (4) may be removed.

Existence theorems without convexity assumptions have been widely investigated in the one-dimensional case $n=1$ (see [12] for an extensive list of references). Theorem 3.1 in Section 3 is specific to the case $n \geq 2$, and it is an extension of some analogous results, obtained under more restrictive assumptions, by Marcellini [22], Mascolo-Schianchi [23], Cellina [7] and Friesecke [21]. In particular, Theorem 3.1 is an extension of related results recently proved by Sychev [28] and Zagatti [29] for integrands independent of $x$ and under a strong assumption on the growth of $f$ which ensures the almost everywhere differentiability of minimizers, i.e., $p>n$, by Celada-Perrotta [5] for $p>1$, and by Dacorogna-Marcellini in [12,13].

Finally we recall that Marcellini [22] pointed out the necessity of the condition of affinity (3) of the function $f^{* *}$ on the set where $f \neq f^{* *}$ to guarantee existence of minimizers. Cellina [6,7] and Friesecke [21] proved the necessity and sufficiency of the condition of affinity for linear boundary data $u_{0}$. The explicit dependence of the integrand on the variable $x$ was first considered by Mascolo-Schianchi in [24], assuming that the divergence of the vector field $m$ in (3) is identically equal to zero in $\Omega$, in addition to other strong assumptions on the boundary data $u_{0}$. Also, in [27] Raymond studied a case where the divergence of the vector field $m$ in (3) is always different from zero in $\Omega$, and some type of explicit dependence on $u$ is allowed.

\section{Local Lipschitz Continuity}

\subsection{Preliminary results}

Let $f: \mathbb{R}^{n} \rightarrow[0,+\infty)$ be a continuous function such that

$$
0 \leq f(\xi) \leq L\left(1+|\xi|^{p}\right)
$$

where $L>0, p>1$. We say that $f$ is uniformly convex at infinity if there exist $R, \nu>0$ such that, if the segment with endpoints $\xi_{1}, \xi_{2}$ (that we will denote by $\left[\xi_{1}, \xi_{2}\right]$ ) is contained in the complement of the closed ball $\bar{B}_{R}$, then

$$
f\left(\frac{\xi_{1}+\xi_{2}}{2}\right) \leq \frac{1}{2} f\left(\xi_{1}\right)+\frac{1}{2} f\left(\xi_{2}\right)-\nu\left(1+\left|\xi_{1}\right|^{2}+\left|\xi_{2}\right|^{2}\right)^{\frac{p-2}{2}}\left|\xi_{1}-\xi_{2}\right|^{2}
$$

Note that (6) is equivalent to

$$
f\left(\frac{\xi_{1}+\xi_{2}}{2}\right) \leq \frac{1}{2} f\left(\xi_{1}\right)+\frac{1}{2} f\left(\xi_{2}\right)-\nu^{\prime}\left(\left|\xi_{1}\right|^{2}+\left|\xi_{2}\right|^{2}\right)^{\frac{p-2}{2}}\left|\xi_{1}-\xi_{2}\right|^{2}
$$

for some $\nu^{\prime}>0$, since $\left|\xi_{1}\right|,\left|\xi_{2}\right|>R>0$.

If the above inequality (6) is satisfied for any $\xi_{1}, \xi_{2} \in \mathbb{R}^{n}$, then we say that $f$ is uniformly convex in $\mathbb{R}^{n}$. A form of uniform convexity at infinity was also considered by Mascolo and Schianchi in [25].

The lemma below is proved in [1].

Lemma 2.1. If $\gamma>-1 / 2$ then there exist positive constants $c_{1}=c_{1}(\gamma), c_{2}=c_{2}(\gamma)$ such that, for all $\xi, \eta \in \mathbb{R}^{n}$,

$$
c_{1}\left(1+|\xi|^{2}+|\eta|^{2}\right)^{\gamma} \leq \int_{0}^{1} t\left(1+|t \xi+(1-t) \eta|^{2}\right)^{\gamma} \mathrm{d} t \leq c_{2}\left(1+|\xi|^{2}+|\eta|^{2}\right)^{\gamma} .
$$

The following result provides two conditions which are equivalent to uniform convexity in $\mathbb{R}^{n}$.

Proposition 2.2. Let $f: \mathbb{R}^{n} \rightarrow[0,+\infty)$ be a continuous function satisfying (5). The following conditions are equivalent:

(i) $f$ is uniformly convex in $\mathbb{R}^{n}$; 
(ii) $f(\xi)=c_{1} \nu\left(1+|\xi|^{2}\right)^{p / 2}+g(\xi)$, for some $c_{1}=c_{1}(p)>0$, where $g(\xi)$ is a convex function such that $0 \leq g(\xi) \leq L\left(1+|\xi|^{p}\right)$ for all $\xi$

(iii) $\int_{Q}[f(\xi+D \varphi(x))-f(\xi)] \mathrm{d} x \geq c_{2} \nu \int_{Q}\left(1+|\xi|^{2}+|D \varphi|^{2}\right)^{\frac{p-2}{2}}|D \varphi(x)|^{2} \mathrm{~d} x$, for all $\xi \in \mathbb{R}^{n}, \varphi \in C_{0}^{1}(Q)$, where $Q=(0,1)^{n}$ and $c_{2}=c_{2}(p)$ is a suitable constant.

Remark 2.3. The condition (iii) in Proposition 2.2 is related to the notion of uniform quasiconvexity, introduced by Evans [16] and later studied by Evans-Gariepy [17].

Proof of Proposition 2.2. (i) $\Longrightarrow$ (ii). We define $g(\xi):=f(\xi)-c_{1} \nu\left(1+|\xi|^{2}\right)^{p / 2}$, where $c_{1}$ will be chosen later, and we show that $g$ is convex. Given $\xi_{1}, \xi_{2} \in \mathbb{R}^{n}$ we set $\xi:=\left(\xi_{1}+\xi_{2}\right) / 2$. From (i) we easily get that

$\frac{1}{2} g\left(\xi_{1}\right)+\frac{1}{2} g\left(\xi_{2}\right) \geq g(\xi)+\nu\left(1+\left|\xi_{1}\right|^{2}+\left|\xi_{2}\right|^{2}\right)^{\frac{p-2}{2}}\left|\xi_{1}-\xi_{2}\right|^{2}+\frac{c_{1} \nu}{2}\left[2\left(1+|\xi|^{2}\right)^{p / 2}-\left(1+\left|\xi_{1}\right|^{2}\right)^{p / 2}-\left(1+\left|\xi_{2}\right|^{2}\right)^{p / 2}\right]$.

Thus the assertion follows immediately from the fact that there exists a constant $c=c(p)$ such that

$$
\left(1+\left|\xi_{1}\right|^{2}\right)^{p / 2}+\left(1+\left|\xi_{2}\right|^{2}\right)^{p / 2}-2\left(1+|\xi|^{2}\right)^{p / 2} \leq c\left(1+\left|\xi_{1}\right|^{2}+\left|\xi_{2}\right|^{2}\right)^{\frac{p-2}{2}}\left|\xi_{1}-\xi_{2}\right|^{2},
$$

and by setting $c_{1}:=c^{-1}$. To establish this inequality we write, for $i=1,2$,

$$
h\left(\xi_{i}\right)=h(\xi)+\left\langle D h(\xi), \xi_{i}-\xi\right\rangle+\int_{0}^{1}(1-t)\left\langle D^{2} h\left(\xi+t\left(\xi_{i}-\xi\right)\right)\left(\xi_{i}-\xi\right), \xi_{i}-\xi\right\rangle \mathrm{d} t
$$

where $h(\xi):=\left(1+|\xi|^{2}\right)^{p / 2}$, yielding

$$
\left(1+\left|\xi_{i}\right|^{2}\right)^{p / 2}-\left(1+|\xi|^{2}\right)^{p / 2} \leq p\left(1+|\xi|^{2}\right)^{\frac{p-2}{2}}\left\langle\xi_{i}-\xi, \xi\right\rangle+c(p)\left|\xi_{i}-\xi\right|^{2} \int_{0}^{1}(1-t)\left(1+\left|\xi+t\left(\xi_{i}-\xi\right)\right|^{2}\right)^{\frac{p-2}{2}} \mathrm{~d} t .
$$

It suffices now to sum the above inequalities for $i=1,2$ and apply Lemma 2.1.

(ii) $\Longrightarrow$ (iii). From Lemma 2.1 we easily get that (iii) holds for the function $\xi \mapsto\left(1+|\xi|^{2}\right)^{p / 2}$. Hence the general case follows from Jensen's inequality applied to $g$.

(iii) $\Longrightarrow$ (i). See the proof of Proposition 2.5 with $\theta=1 / 2$ in [19].

Lemma 2.4. Let $f: \mathbb{R}^{n} \rightarrow[0,+\infty)$ be a $C^{2}$ function. Then $f$ satisfies (6) if and only if there exists a constant $c_{0}$ such that for all $\xi \in \mathbb{R}^{n} \backslash \bar{B}_{R}$

$$
\left\langle D^{2} f(\xi) \lambda, \lambda\right\rangle \geq c_{0} \nu\left(1+|\xi|^{2}\right)^{\frac{p-2}{2}}|\lambda|^{2} \quad \forall \lambda \in \mathbb{R}^{n} .
$$

The proof of Lemma 2.4 is straightforward and it is left to the reader.

Lemma 2.5. Let $f: \mathbb{R}^{n} \rightarrow[0,+\infty)$ be a continuous function satisfying (5) and (6). Then there exist $R_{0}, \nu_{0}, C_{0}>0$, depending only on $R, \nu$ and $L$, such that for all $\xi \in \mathbb{R}^{n} \backslash \bar{B}_{R_{0}}$ there exists $q_{\xi} \in \mathbb{R}^{n}$ such that $\left|q_{\xi}\right| \leq C_{0}\left(1+|\xi|^{p-1}\right)$ and

$$
f(\eta) \geq f(\xi)+\left\langle q_{\xi}, \eta-\xi\right\rangle+\nu_{0}\left(1+|\xi|^{2}+|\eta|^{2}\right)^{\frac{p-2}{2}}|\xi-\eta|^{2} \quad \forall \eta \in \mathbb{R}^{n} .
$$

Moreover, if $|\xi|>R_{0}$, then $f^{* *}(\xi)=f(\xi)$.

Proof. For $0<\varepsilon<1$ set $f_{\varepsilon}:=\rho_{\varepsilon} * f$, where $\rho_{\varepsilon}(\eta):=\varepsilon^{-n} \rho(\eta / \varepsilon)$ and $\rho(\eta)=\hat{\rho}(|\eta|)$ is a positive radially symmetric mollifier with support equal to $\bar{B}$, with $B:=B_{1}, \rho(\eta)>0$ if $|\eta|<1$ and $\int_{B} \rho(\eta) \mathrm{d} \eta=1$. From (6) it follows easily that if $\left[\xi_{1}, \xi_{2}\right] \subset \mathbb{R}^{n} \backslash \bar{B}_{R+1}, \xi:=\left(\xi_{1}+\xi_{2}\right) / 2$, then

$$
\frac{1}{2}\left[f_{\varepsilon}\left(\xi_{1}\right)+f_{\varepsilon}\left(\xi_{2}\right)\right] \geq f_{\varepsilon}(\xi)+\nu\left|\xi_{1}-\xi_{2}\right|^{2} \int_{B} \rho(\eta)\left(1+\left|\xi_{1}+\varepsilon \eta\right|^{2}+\left|\xi_{2}+\varepsilon \eta\right|^{2}\right)^{\frac{p-2}{2}} \mathrm{~d} \eta .
$$


The integral above can be estimated from below by

$$
\int_{B \cap\{\langle\xi, \eta\rangle \geq 0\}} \rho(\eta)\left(1+\left|\xi_{1}\right|^{2}+\left|\xi_{2}\right|^{2}+2 \varepsilon^{2}|\eta|^{2}+4 \varepsilon\langle\xi, \eta\rangle\right)^{\frac{p-2}{2}} \mathrm{~d} \eta \geq \frac{1}{2}\left(1+\left|\xi_{1}\right|^{2}+\left|\xi_{2}\right|^{2}\right)^{\frac{p-2}{2}}
$$

if $p \geq 2$, and by

$$
\int_{B} \rho(\eta)\left(1+2\left|\xi_{1}\right|^{2}+2\left|\xi_{2}\right|^{2}+4 \varepsilon^{2}|\eta|^{2}\right)^{\frac{p-2}{2}} \mathrm{~d} \eta \geq 5^{(p-2) / 2}\left(1+\left|\xi_{1}\right|^{2}+\left|\xi_{2}\right|^{2}\right)^{\frac{p-2}{2}}
$$

when $1<p<2$. In both cases

$$
\frac{1}{2}\left[f_{\varepsilon}\left(\xi_{1}\right)+f_{\varepsilon}\left(\xi_{2}\right)\right] \geq f_{\varepsilon}(\xi)+c \nu\left(1+\left|\xi_{1}\right|^{2}+\left|\xi_{2}\right|^{2}\right)^{\frac{p-2}{2}}\left|\xi_{1}-\xi_{2}\right|^{2}
$$

hence, by Lemma 2.4 , if $|\xi| \geq R+1$ then

$$
\left\langle D^{2} f_{\varepsilon}(\xi) \lambda, \lambda\right\rangle \geq c \nu\left(1+|\xi|^{2}\right)^{\frac{p-2}{2}}|\lambda|^{2}, \quad \forall \lambda \in \mathbb{R}^{n} .
$$

Moreover, it can be easily checked that $0 \leq f_{\varepsilon}(\xi) \leq C(L)\left(1+|\xi|^{p}\right)$ for all $\xi$, and a simple argument based on the convexity of $f_{\varepsilon}$ in $\mathbb{R}^{n} \backslash \bar{B}_{R+1}$ shows that there exists a constant $C_{1}(L, R)$ such that

$$
\left|D f_{\varepsilon}(\xi)\right| \leq C_{1}\left(1+|\xi|^{p-1}\right), \quad \forall \xi \in \mathbb{R}^{n} \backslash \bar{B}_{R+2}
$$

We claim that there exists $R_{0} \gg 1$ such that if $|\xi|>R_{0}$ then

$$
f_{\varepsilon}(\eta) \geq f_{\varepsilon}(\xi)+\left\langle D f_{\varepsilon}(\xi), \eta-\xi\right\rangle+c\left(1+|\xi|^{2}+|\eta|^{2}\right)^{\frac{p-2}{2}}|\xi-\eta|^{2}
$$

for all $\eta \in \mathbb{R}^{n}$. Assume that (11) holds. Notice that, if $|\xi|>R_{0}>R+2$, then by (10) there exists a sequence $\left(\varepsilon_{h}\right)$ converging to 0 such that $D f_{\varepsilon_{h}}(\xi) \rightarrow q_{\xi}$ for some $q_{\xi} \in \mathbb{R}^{n}$ such that $\left|q_{\xi}\right| \leq C_{1}\left(1+|\xi|^{p-1}\right)$. Hence (8) follows from (11), letting $\varepsilon$ go to $0^{+}$. The equality $f^{* *}(\xi)=f(\xi)$ for $|\xi|>R_{0}$ then follows at once from (8).

The remaining of the proof concerns the assertion of the claim (11). Fix $\xi$ such that $|\xi|>R_{0}$, with $R_{0}>$ $2(R+3)$ to be chosen later, and denote by $C_{\xi}$ the open cone with vertex at $\xi$, tangent to the ball $\bar{B}_{R+3}$.

Case 1: If $\eta \in \mathbb{R}^{n} \backslash C_{\xi}$ or $\eta \in C_{\xi} \backslash B_{R+3}$ and $\langle\eta, \xi\rangle \geq 0$, then $f_{\varepsilon}$ is convex along the line $t \rightarrow \xi+t(\eta-\xi)$ provided $R_{0}$ is sufficiently large, and (11) follows from (9).

Case 2: If $\eta \in B_{R+3}$ we consider $\bar{\xi}:=\xi(R+3) /|\xi|$ and a constant $M:=C(L)\left(1+(R+3)^{p}\right)$ such that

$$
0 \leq f_{\varepsilon}(\eta) \leq M
$$

for all $\eta \in B_{R+3}$ and all $\varepsilon \in(0,1)$; by Case 1 we may apply (11) to $\bar{\xi}$ (notice that $\bar{\xi} \in \partial B_{R+3},\langle\bar{\xi}, \xi\rangle>0$ ), thus getting

$$
\begin{gathered}
f_{\varepsilon}(\eta)=f_{\varepsilon}(\bar{\xi})+f_{\varepsilon}(\eta)-f_{\varepsilon}(\bar{\xi}) \\
\geq f_{\varepsilon}(\xi)+\left\langle D f_{\varepsilon}(\xi), \bar{\xi}-\xi\right\rangle-M+c\left(1+(R+3)^{2}+|\xi|^{2}\right)^{\frac{p-2}{2}}(|\xi|-R-3)^{2} \\
\geq f_{\varepsilon}(\xi)+\left\langle D f_{\varepsilon}(\xi), \eta-\xi\right\rangle-\left|D f_{\varepsilon}(\xi)\right||\eta-\bar{\xi}|-M+\tilde{c}\left(1+|\eta|^{2}+|\xi|^{2}\right)^{\frac{p-2}{2}}|\xi-\eta|^{2} .
\end{gathered}
$$


The estimate (11) now follows for $\eta$ and $\xi$ from the previous inequalities, together with the estimate

$$
\left|D f_{\varepsilon}(\xi)\right||\eta-\bar{\xi}|+M \leq \frac{1}{2} \tilde{c}\left(1+|\eta|^{2}+|\xi|^{2}\right)^{\frac{p-2}{2}}|\xi-\eta|^{2},
$$

and the latter holds by virtue of (10), and provided $\xi>R_{0}$ and $R_{0}>2(R+3)$ are sufficiently large.

Case 3: Finally, let us assume that $\eta \in C_{\xi} \backslash B_{R+3},\langle\eta, \xi\rangle<0$. In this case we have $|\xi-\eta|^{2}>|\xi|^{2}+|\eta|^{2}$, and denoting by $\tilde{\eta}$ the projection of $\eta$ on the boundary of the cone, and by $\alpha_{\xi}$ the half angle at the vertex of $C_{\xi}$,

$$
|\eta-\tilde{\eta}| \leq|\xi-\eta| \sin \alpha_{\xi}=\frac{R+3}{|\xi|}|\xi-\eta| \leq \frac{1}{2}|\xi-\eta| .
$$

Notice that if $R_{0}$ is sufficiently large then $[\eta, \tilde{\eta}] \subset \mathbb{R}^{n} \backslash \bar{B}_{R+2}$; therefore we may use (10) to estimate $f_{\varepsilon}(\eta)-f_{\varepsilon}(\tilde{\eta})$. This, together with (11) applied to $\tilde{\eta} \in \partial C_{\xi}$, yields

$$
\begin{aligned}
f_{\varepsilon}(\eta)=f_{\varepsilon}(\tilde{\eta})+f_{\varepsilon}(\eta)-f_{\varepsilon}(\tilde{\eta}) \geq & f_{\varepsilon}(\xi)+\left\langle D f_{\varepsilon}(\xi), \tilde{\eta}-\xi\right\rangle-C_{1}\left(1+|\eta|^{p-1}+|\tilde{\eta}|^{p-1}\right)|\eta-\tilde{\eta}| \\
& +c\left(1+|\tilde{\eta}|^{2}+|\xi|^{2}\right)^{\frac{p-2}{2}}|\xi-\tilde{\eta}|^{2} .
\end{aligned}
$$

Since, by (12), $|\xi-\eta| \leq 2|\xi-\tilde{\eta}| \leq 3|\xi-\eta|$, for any $p>1$ we have easily

$$
c\left(1+|\tilde{\eta}|^{2}+|\xi|^{2}\right)^{\frac{p-2}{2}}|\xi-\tilde{\eta}|^{2} \geq \bar{c}(p)\left(1+|\eta|^{2}+|\xi|^{2}\right)^{\frac{p-2}{2}}|\xi-\eta|^{2},
$$

and, using (10) once more, we obtain

$$
\begin{aligned}
f_{\varepsilon}(\eta) \geq & f_{\varepsilon}(\xi)+\left\langle D f_{\varepsilon}(\xi), \eta-\xi\right\rangle-C_{1}|\eta-\tilde{\eta}|\left[\left(1+|\xi|^{p-1}\right)+\left(1+|\eta|^{p-1}+|\tilde{\eta}|^{p-1}\right)\right] \\
& +\bar{c}(p)\left(1+|\eta|^{2}+|\xi|^{2}\right)^{\frac{p-2}{2}}|\xi-\eta|^{2}
\end{aligned}
$$

By virtue of (12), and recalling that $|\xi|^{2}+|\eta|^{2}<|\xi-\eta|^{2}$, we have

$$
\begin{aligned}
C_{1}|\eta-\tilde{\eta}|\left[\left(1+|\xi|^{p-1}\right)+\left(1+|\eta|^{p-1}+|\tilde{\eta}|^{p-1}\right)\right] & \leq c \frac{R+3}{|\xi|}|\xi-\eta|\left(1+|\eta|^{p-1}+|\xi|^{p-1}\right) \\
& \leq c^{\prime} \frac{R+3}{|\xi|}|\xi-\eta|^{2}\left(1+|\eta|^{2}+|\xi|^{2}\right)^{\frac{p-2}{2}} \\
& \leq(\bar{c}(p) / 2)\left(1+|\eta|^{2}+|\xi|^{2}\right)^{\frac{p-2}{2}}|\xi-\eta|^{2}
\end{aligned}
$$

if $|\xi|>R_{0}$, with $R_{0}$ large enough. This, together with (13), concludes the proof of (11).

Remark 2.6. Let $f$ satisfy (5) and (6), and fix a point $\xi_{0}$ such that $R_{0}<\left|\xi_{0}\right|<2 R_{0}$. Applying (8) with $\xi=\xi_{0}$ and recalling that $\left|q_{\xi}\right| \leq C_{0}\left(1+|\xi|^{p-1}\right)$, for all $\eta$ such that $|\eta|>2 R_{0}$ it holds

$$
f(\eta) \geq \tilde{c}_{1}\left(R_{0}, \nu_{0}, C_{0}\right)|\eta|^{p}-\tilde{c}_{2}\left(R_{0}, \nu_{0}, C_{0}\right) .
$$

Hence, $f(\xi) \geq c_{1}|\xi|^{p}-c_{2}$ for all $\xi \in \mathbb{R}^{n}$, with $c_{1}, c_{2}$ depending only on $R, \nu, L$.

\subsection{A regularity result}

In this section we assume that $f: \Omega \times \mathbb{R}^{n} \rightarrow[0,+\infty)$ is a continuous function satisfying the growth condition

$$
0 \leq f(x, \xi) \leq L\left(1+|\xi|^{p}\right), \quad \forall(x, \xi) \in \Omega \times \mathbb{R}^{n}
$$


and for some $L>0$. Let us denote by $f^{* *}:=f^{* *}(x, \xi)$ the bipolar of $f$, that is the convex envelope of $f(x, \cdot)$. We assume that $f^{* *}$ is continuous and that $f$ is uniformly convex at infinity with respect to $\xi$ (see (6)), i.e., there exist $R, \nu>0$ such that if the segment $\xi_{1}, \xi_{2}$ is contained in the complement of the closed ball $\bar{B}_{R}$, then for all $x \in \Omega$

$$
f\left(x, \frac{\xi_{1}+\xi_{2}}{2}\right) \leq \frac{1}{2} f\left(x, \xi_{1}\right)+\frac{1}{2} f\left(x, \xi_{2}\right)-\nu\left(1+\left|\xi_{1}\right|^{2}+\left|\xi_{2}\right|^{2}\right)^{\frac{p-2}{2}}\left|\xi_{1}-\xi_{2}\right|^{2}
$$

Finally we assume further that if $|\xi|>R$ then the vector field $x \in \Omega \mapsto f_{\xi}(x, \xi)$ is weakly differentiable and

$$
\left|D_{x} f_{\xi}(x, \xi)\right| \leq L\left(1+|\xi|^{p-1}\right), \quad \forall(x, \xi) \in \Omega \times\left(\mathbb{R}^{n} \backslash \bar{B}_{R}\right) .
$$

If $u \in W_{\mathrm{loc}}^{1, p}(\Omega)$ and $A \subset \Omega$ is open, then we set

$$
F(u, A):=\int_{A} f(x, D u(x)) \mathrm{d} x .
$$

The main result of this section is Theorem 2.7 below. We recall that $u$ is said to be a local minimizer of $F$ in $\Omega$ if

$$
F\left(u, B_{R}\left(x_{0}\right)\right) \leq F\left(v, B_{R}\left(x_{0}\right)\right)
$$

whenever $B_{R}\left(x_{0}\right) \subset \subset \Omega$ and $v \in u+W_{0}^{1, p}\left(B_{R}\left(x_{0}\right)\right)$.

Theorem 2.7. Let $f: \Omega \times \mathbb{R}^{n} \rightarrow[0,+\infty)$ be a continuous function satisfying $(14,15)$ and $(16)$. If $u \in W_{\text {loc }}^{1, p}(\Omega)$ is a local minimizer of the functional $F$, then $u$ is locally Lipschitz continuous in $\Omega$. Moreover, there exists a constant $C_{0}$, depending on $L, p, \nu, R$, such that if $B_{r}\left(x_{0}\right) \subset \Omega$ then

$$
\sup _{B_{r / 2}\left(x_{0}\right)}|D u|^{p} \leq C_{0}\left(1+f_{B_{r}\left(x_{0}\right)}|D u|^{p} \mathrm{~d} x\right) .
$$

We first show in Lemma 2.8 that, provided we know already that $u$ is locally Lipschitz, equation (17) holds with a constant $C_{0}$ depending only on $L, p, \nu, R$. Once the a priori estimate (17) is established, the regularity result is obtained using an approximation argument.

Lemma 2.8. Let $f$ satisfy the assumptions of Theorem 2.\%. Assume, in addition, that $f$ is $C^{2}$ and that, for all $x \in \Omega, \xi, \lambda \in \mathbb{R}^{n}$,

$$
D_{\xi_{i} \xi_{j}} f(x, \xi) \lambda_{i} \lambda_{j} \geq \varepsilon_{0}\left(1+|\xi|^{2}\right)^{\frac{p-2}{2}}|\lambda|^{2}
$$

and that $u \in W_{\mathrm{loc}}^{1, p}(\Omega)$ is a locally Lipschitz local minimizer of $F$ in $\Omega$. Then (17) holds with a constant depending only on $L, p, \nu, R$ and, in particular, independent on $\varepsilon_{0}$.

Proof. Step 1: From Lemma 2.4 we have that for every $x \in \Omega, \xi, \lambda \in \mathbb{R}^{n}$, with $|\xi|>R$,

$$
D_{\xi_{i} \xi_{j}} f(x, \xi) \lambda_{i} \lambda_{j} \geq c \nu\left(1+|\xi|^{2}\right)^{\frac{p-2}{2}}|\lambda|^{2} .
$$

Since $\tilde{u}(y):=u\left(x_{0}+r y\right) / r$ is a local minimizer in $\left(\Omega-x_{0}\right) / r$ of the functional $\tilde{F}$, where $\tilde{F}(v):=$ $\int_{\left(\Omega-x_{0}\right) / r} f\left(x_{0}+r y, D v(y)\right) \mathrm{d} y$ still satisfies the assumptions of Theorem 2.7, it is clear that in order to prove (17) we may always assume, with no loss of generality, that $B:=B_{1} \subset \Omega$. Since $u$ satisfies the Euler equation for $F$,

$$
\int_{B} D_{\xi_{i}} f(x, D u) D_{i} \phi \mathrm{d} x=0, \quad \forall \phi \in C_{0}^{1}(B),
$$


using (18) and the fact that $D_{\xi_{i} \xi_{j}} f(x, D u(x))$ are locally bounded in $B$ (which follows from the $C^{2}$ regularity of $f$, together with the fact that $D u$ is locally bounded) we have that $u \in W_{\text {loc }}^{2,2}(B)$, by a standard different quotient argument.

We fix $s \in\{1, \ldots, n\}, \eta \in C_{0}^{1}(B), 0 \leq \eta \leq 1, \psi \in C^{2}(B)$, and in the above Euler equation we take $\phi=\eta^{2} D_{s} \psi$ to obtain

$$
\int_{B} D_{\xi_{i}} f(x, D u) D_{s}\left(D_{i} \psi\right) \eta^{2} \mathrm{~d} x=-2 \int_{B} \eta D_{\xi_{i}} f(x, D u) D_{s} \psi D_{i} \eta \mathrm{d} x .
$$

Integrating by parts the first integral, we have

$$
\begin{aligned}
\int_{B} D_{\xi_{i} \xi_{j}} f(x, D u) D_{j}\left(D_{s} u\right) D_{i} \psi \eta^{2} \mathrm{~d} x= & 2 \int_{B} \eta D_{\xi_{i}} f(x, D u) D_{s} \psi D_{i} \eta \mathrm{d} x \\
& -2 \int_{B} D_{x_{s} \xi_{i}} f(x, D u) D_{i} \psi \eta^{2} \mathrm{~d} x-2 \int_{B} \eta D_{\xi_{i}} f(x, D u) D_{i} \psi D_{s} \eta \mathrm{d} x
\end{aligned}
$$

for all functions $\psi \in W^{1,2}(B)$. Set

$$
V_{+}(x):=1+R^{2}+\sum_{h=1}^{n}\left[\left(D_{h} u(x)-R\right)^{+}\right]^{2}, \quad V_{-}(x):=1+R^{2}+\sum_{h=1}^{n}\left[\left(D_{h} u(x)+R\right)^{-}\right]^{2},
$$

and notice that there exist constants $c_{1}, c_{2}$, depending only on $n$, such that

$$
c_{1}\left(V_{+}(x)+V_{-}(x)\right) \leq 1+R^{2}+|D u(x)|^{2} \leq c_{2}\left(V_{+}(x)+V_{-}(x)\right) .
$$

Let $\psi:=V_{+}^{\beta}\left(D_{s} u-R\right)^{+}$, where $\beta \geq 0$. By (14) and the convexity of $f(x, \cdot)$ we get $\left|D_{\xi} f(x, \xi)\right| \leq c\left(1+|\xi|^{2}\right)^{\frac{p-1}{2}}$; this inequality, together with (16) and (20), yields

$$
\begin{aligned}
& \int_{B} D_{\xi_{i} \xi_{j}} f(x, D u) D_{j}\left(D_{s} u-R\right)^{+} D_{i}\left(D_{s} u-R\right)^{+} V_{+}^{\beta} \eta^{2} \mathrm{~d} x \\
&+\beta \int_{B} D_{\xi_{i} \xi_{j}} f(x, D u) D_{j}\left(D_{s} u-R\right)^{+}\left(D_{s} u-R\right)^{+} D_{i}\left(\sum_{h=1}^{n}\left[\left(D_{h} u-R\right)^{+}\right]^{2}\right) V_{+}^{\beta-1} \eta^{2} \mathrm{~d} x \\
& \leq c \int_{B} \eta(\eta+|D \eta|)\left(1+|D u|^{p-1}\right)\left|D\left(D_{s} u-R\right)^{+}\right| V_{+}^{\beta} \mathrm{d} x \\
& \quad+c \beta \int_{B} \eta(\eta+|D \eta|)\left(1+|D u|^{p-1}\right)\left|D\left(\sum_{h=1}^{n}\left[\left(D_{h} u-R\right)^{+}\right]^{2}\right)\right|\left(D_{s} u-R\right)^{+} V_{+}^{\beta-1} \mathrm{~d} x .
\end{aligned}
$$

Since all the integrals are evaluated in the set where $|D u|>R$, summing up on $s$, using (19), the fact that $\left(D_{s} u-R\right)^{+} \leq V_{+}^{1 / 2}$ and Young's inequality, it follows easily that

$$
\int_{B}\left(1+|D u|^{2}\right)^{\frac{p-2}{2}}\left|D\left(\sum_{h=1}^{n}\left[\left(D_{h} u-R\right)^{+}\right]^{2}\right)\right|^{2} V_{+}^{\beta-1} \eta^{2} \mathrm{~d} x \leq \frac{c}{\nu^{2}} \int_{B}\left(1+|D u|^{p}\right) V_{+}^{\beta}\left(\eta^{2}+|D \eta|^{2}\right) \mathrm{d} x,
$$

where the constant $c$ depends only on $n, p, L$. Since the integral on the left hand side is evaluated in the set where $|D u|>R$, in turn this last inequality is equivalent to the following one:

$$
\int_{B}\left(1+R^{2}+|D u|^{2}\right)^{\frac{p-2}{2}}\left|D\left(\sum_{h=1}^{n}\left[\left(D_{h} u-R\right)^{+}\right]^{2}\right)\right|^{2} V_{+}^{\beta-1} \eta^{2} \mathrm{~d} x \leq \frac{c}{\nu^{2}} \int_{B}\left(1+R^{2}+|D u|^{2}\right)^{p / 2} V_{+}^{\beta}\left(\eta^{2}+|D \eta|^{2}\right) \mathrm{d} x .
$$


Inserting $\psi=V_{-}^{\beta}\left(D_{s} u+R\right)^{-}$in (20), and using a similar argument, we get also

$$
\int_{B}\left(1+R^{2}+|D u|^{2}\right)^{\frac{p-2}{2}}\left|D\left(\sum_{h=1}^{n}\left[\left(D_{i} u+R\right)^{-}\right]^{2}\right)\right|^{2} V_{-}^{\beta-1} \eta^{2} \mathrm{~d} x \leq \frac{c}{\nu^{2}} \int_{B}\left(1+R^{2}+|D u|^{2}\right)^{p / 2} V_{-}^{\beta}\left(\eta^{2}+|D \eta|^{2}\right) \mathrm{d} x .
$$

Therefore, adding the last two inequalities and using (21), we arrive to

$$
\int_{B} V^{\frac{p-2}{2}}\left[\left|D V_{+}\right|^{2} V_{+}^{\beta-1}+\left|D V_{-}\right|^{2} V_{-}^{\beta-1}\right] \eta^{2} \mathrm{~d} x \leq \frac{c}{\nu^{2}} \int_{B} V^{p / 2+\beta}\left(\eta^{2}+|D \eta|^{2}\right) \mathrm{d} x
$$

where $V:=\max \left\{V_{+} ; V_{-}\right\}$.

Step 2: From the inequality above we deduce that

$$
\int_{B}\left|D V^{p / 4+\beta / 2}\right|^{2} \eta^{2} \mathrm{~d} x \leq c(p+2 \beta)^{2} \int_{B} V^{p / 2+\beta}\left(\eta^{2}+|D \eta|^{2}\right) \mathrm{d} x .
$$

In turn, this implies that

$$
\int_{B}\left|D\left(V^{p / 4+\beta / 2} \eta\right)\right|^{2} \mathrm{~d} x \leq c(p+2 \beta)^{2} \int_{B} V^{p / 2+\beta}\left(\eta^{2}+|D \eta|^{2}\right) \mathrm{d} x,
$$

where the constant $c$ depends only on $L, p, n, R, \nu$. Setting $\gamma:=p / 4+\beta / 2 \geq p / 4$, using the Sobolev-Poincaré inequality, and the arbitrariness of $\beta \geq 0$, we get that, for any $\gamma \geq p / 4$,

$$
\left\|V^{\gamma} \eta\right\|_{L^{2 \chi}(B)} \leq c \gamma\left\|V^{\gamma}(\eta+|D \eta|)\right\|_{L^{2}(B)},
$$

where $\chi:=n /(n-2)$ if $n \geq 3$, or any number $>1$ if $n=2$. Considering the sequence of radii $r_{i}:=1 / 2+1 / 2^{i}$ for $i=1, \ldots$, we apply the inequality above to $\gamma=\gamma_{i}:=(p / 4) \chi^{i-1}$, and choose $\eta \in C_{0}^{1}(B)$ such that $\eta=1$ on $B_{r_{i+1}}, 0 \leq \eta \leq 1,|D \eta| \leq c 2^{i}$. We obtain

$$
\|V\|_{L^{2 \gamma_{i+1}\left(B_{r_{i+1}}\right)}} \leq\left(c 2^{i} \gamma_{i}\right)^{1 / \gamma_{i}}\|V\|_{L^{2 \gamma_{i}\left(B_{r_{i}}\right)}} .
$$

Iterating the above formula yields, for every $i$,

$$
\|V\|_{L^{2 \gamma_{i+1}\left(B_{1 / 2}\right)}} \leq C\|V\|_{L^{p / 2}(B)},
$$

where $C=\prod_{i=1}^{\infty}\left(c 2^{i} \gamma_{i}\right)^{1 / \gamma_{i}}<+\infty$. Therefore, letting $i$ go to $+\infty$ and using (21), we obtain (17).

Remark 2.9. It follows immediately from the proof that the estimate (17) may be generalized to read

$$
\sup _{B_{\rho}\left(x_{0}\right)}|D u|^{p} \leq C(\rho)\left(1+f_{B_{r}\left(x_{0}\right)}|D u|^{p} \mathrm{~d} x\right),
$$

for all $0<\rho<r$, where $C(\rho)$ depends only on $L, p, \nu, R$ and $\rho$.

We are now in position to prove Theorem 2.7 , by using the following approximation lemma.

Lemma 2.10. Let $g: \mathbb{R}^{n} \rightarrow[0,+\infty)$ be a $C^{2}$ convex function such that for all $\xi \in \mathbb{R}^{n}$

$$
0 \leq g(\xi) \leq L\left(1+|\xi|^{p}\right),
$$


where $p>1, L>0$, and assume that there exist $R, \nu>0$, such that if $|\xi|>R, \lambda \in \mathbb{R}^{n}$,

$$
D_{i j} g(\xi) \lambda_{i} \lambda_{j} \geq \nu\left(1+|\xi|^{2}\right)^{\frac{p-2}{2}}|\lambda|^{2} .
$$

Then there exists a constant $c=c(n, p)$ and a sequence $g_{h}$ of $C^{2}\left(\mathbb{R}^{n}\right)$ convex functions such that

(a) $0 \leq g_{h}(\xi) \leq c L\left(1+|\xi|^{p}\right) \quad \forall \xi \in \mathbb{R}^{n}$;

(b) for any $h$ there exists $\varepsilon_{h}>0$ such that, for all $\xi, \lambda \in \mathbb{R}^{n}$,

$$
\varepsilon_{h}\left(1+|\xi|^{2}\right)^{\frac{p-2}{2}}|\lambda|^{2} \leq D_{i j} g_{h}(\xi) \lambda_{i} \lambda_{j} \leq \varepsilon_{h}^{-1}\left(1+|\xi|^{2}\right)^{\frac{p-2}{2}}|\lambda|^{2}
$$

(c) $D_{i j} g_{h}(\xi) \lambda_{i} \lambda_{j} \geq c \nu\left(1+|\xi|^{2}\right)^{\frac{p-2}{2}}|\lambda|^{2}, \forall \lambda \in \mathbb{R}^{n},|\xi|>R+1$;

(d) $g_{h} \rightarrow g$ uniformly on compact subset of $\mathbb{R}^{n}$.

Proof. The proof of this lemma can be obtained arguing as in the Step 2 and Step 3 of the proof of Lemma 3.4 of [19], with the obvious simple modifications needed in the present case. Therefore we omit the details.

Proof of Theorem 2.7. Notice that if $u$ is a local minimizer of $F$, then $u$ is also a local minimizer of the relaxed functional $v \mapsto \int_{\Omega} f^{* *}(x, D v) \mathrm{d} x$, where, for all $x \in \Omega, f^{* *}(x, \xi)$ is the bipolar of $\xi \mapsto f(x, \xi)$. Indeed, if $v \in u+W_{0}^{1, p}\left(B_{r}\left(x_{0}\right)\right)$ then

$$
\int_{B_{r}\left(x_{0}\right)} f^{* *}(x, D v) \mathrm{d} x=\inf \left\{\liminf _{h \rightarrow+\infty} \int_{B_{r}\left(x_{0}\right)} f\left(x, D v_{h}\right) \mathrm{d} x: v_{h}-v \rightarrow 0 \text { in } w-W_{0}^{1, p}\left(B_{r}\left(x_{0}\right)\right)\right\} .
$$

Also, by virtue of Lemma 2.5, the function $f^{* *}$ satisfies the assumptions of Theorem 2.7. Therefore, with no loss of generality, we may assume that $f$ is convex in $\xi$.

Step 1: Let us assume that

$$
f(x, \xi)=\sum_{i=1}^{N} a_{i}(x) g_{i}(\xi),
$$

where, for $i=1, \ldots, N$, the function $g_{i} \in C^{2}\left(\mathbb{R}^{n}\right)$ satisfies the assumptions of Lemma 2.10 for some $L, R, \nu>0$, and $\left\langle D^{2} g_{i}(\xi) \lambda, \lambda\right\rangle \geq \varepsilon_{0}\left(1+|\xi|^{2}\right)^{(p-2) / 2}|\lambda|^{2}$ for all $\xi, \lambda \in \mathbb{R}^{n}$ and for some $\varepsilon_{0}>0$. Moreover, let us assume that, for all $i$, the function $a_{i}$ is a nonnegative $C^{2}$ function such that $\left\|D a_{i}\right\|_{\infty} \leq M$ and that $\gamma^{-1}<\sum_{i=1}^{N} a_{i}(x)<\gamma$ for all $x \in \Omega$ and for some positive constant $\gamma$.

For every $i$ let us denote by $g_{i, h}$ a sequence of $C^{2}\left(\mathbb{R}^{n}\right)$ functions such that $g_{i, h} \rightarrow g_{i}$ uniformly on the compact sets of $\mathbb{R}^{n}$, satisfying the conditions $(a, b)$ and $(c)$ of Lemma 2.10 , and let us set for all $(x, \xi) \in \Omega \times \mathbb{R}^{n}$

$$
f_{h}(x, \xi):=\sum_{i=1}^{N} a_{i}(x) g_{i, h}(\xi) .
$$

From Remark 2.6 it follows easily that there exist constants $c_{1}, c_{2}$, depending only on $L, R, \nu, \gamma$, such that, for all $(x, \xi)$ and for any $h$,

$$
f(x, \xi), f_{h}(x, \xi) \geq c_{1}|\xi|^{p}-c_{2} .
$$

Given $B_{r}\left(x_{0}\right) \subset \subset \Omega$, we denote by $u_{h}$ the solution of the problem

$$
\min \left\{\int_{B_{r}\left(x_{0}\right)} f_{h}(x, D v) \mathrm{d} x: v \in u+W_{0}^{1, p}\left(B_{r}\left(x_{0}\right)\right)\right\} .
$$


Since the functions $g_{i, h}$ satisfy condition $(b)$ of Lemma 2.10, standard elliptic regularity theory implies that $u_{h} \in C^{1, \alpha}(\Omega) \cap W_{\text {loc }}^{2,2}(\Omega)$ for any $h$. From the assumptions on $f$, from the approximation provided by Lemma 2.10, and from (22), it follows that the sequence $u_{h}$ is bounded in $W^{1, p}\left(B_{r}\left(x_{0}\right)\right.$ ). Moreover, by Lemma 2.8 (see Rem. 2.9), for all $\rho<r$ we obtain

$$
\sup _{B_{\rho}\left(x_{0}\right)}\left|D u_{h}\right|^{p} \leq C\left(1+f_{B_{r}\left(x_{0}\right)}\left|D u_{h}\right|^{p} \mathrm{~d} x\right),
$$

where the constant $C$ depends ultimately only on $L, p, R, \nu, \gamma, M$ and $\rho$, but not on $h$. Hence we may assume, up to a subsequence, that $u_{h} \rightarrow u_{\infty}$ weakly* in $W^{1, \infty}\left(B_{\rho}\left(x_{0}\right)\right)$ for any $\rho<r$. Since $f_{h} \rightarrow f$ uniformly on compact sets of $\mathbb{R}^{n}$ and the integrand $f$ is convex, for any $\rho<r$ we have

$$
\begin{aligned}
\int_{B_{\rho}\left(x_{0}\right)} f\left(x, D u_{\infty}\right) \mathrm{d} x & \leq \liminf _{h \rightarrow+\infty} \int_{B_{\rho}\left(x_{0}\right)} f\left(x, D u_{h}\right) \mathrm{d} x=\liminf _{h \rightarrow+\infty} \int_{B_{\rho}\left(x_{0}\right)} f_{h}\left(x, D u_{h}\right) \mathrm{d} x \\
& \leq \liminf _{h \rightarrow+\infty} \int_{B_{r}\left(x_{0}\right)} f_{h}(x, D u) \mathrm{d} x=\int_{B_{r}\left(x_{0}\right)} f(x, D u) \mathrm{d} x,
\end{aligned}
$$

where we used the fact that $u_{h}$ is a solution for (23). Letting $\rho \rightarrow r$ and recalling that $u$ is a local minimizer of the functional $F$, we deduce that $u_{\infty}$ is also a minimizer of $F$ in $B_{r}\left(x_{0}\right)$. Since the functional $F$ is strictly convex, we have that $u=u_{\infty}$. Passing to the limit as $h \rightarrow+\infty$ in (24), we conclude that $u$ is also locally Lipschitz. Indeed, using the minimality of $u_{h}$ and (24), we get

$$
\begin{aligned}
\sup _{B_{r / 2}\left(x_{0}\right)}|D u|^{p} & \leq \liminf _{h \rightarrow+\infty}\left(\sup _{B_{r / 2}\left(x_{0}\right)}\left|D u_{h}\right|^{p}\right) \\
& \leq c \liminf _{h \rightarrow+\infty}\left(1+f_{B_{r}\left(x_{0}\right)}\left|D u_{h}\right|^{p} \mathrm{~d} x\right) \leq c \liminf _{h \rightarrow+\infty}\left(1+f_{B_{r}\left(x_{0}\right)} f_{h}\left(x, D u_{h}\right) \mathrm{d} x\right) \\
& \leq c \liminf _{h \rightarrow+\infty}\left(1+f_{B_{r}\left(x_{0}\right)} f_{h}(x, D u) \mathrm{d} x\right) \leq c\left(1+f_{B_{r}\left(x_{0}\right)}|D u|^{p} \mathrm{~d} x\right) .
\end{aligned}
$$

Step 2: Let us now assume that $f \in C^{2}\left(\Omega \times \mathbb{R}^{n}\right)$ and that there exists $\varepsilon_{0}>0$ such that $D_{\xi_{i} \xi_{j}} f(x, \xi) \lambda_{i} \lambda_{j} \geq$ $\varepsilon_{0}\left(1+|\xi|^{2}\right)^{(p-2) / 2}|\lambda|^{2}$ for all $(x, \xi) \in \Omega \times \mathbb{R}^{n}$ and for any $\lambda \in \mathbb{R}^{n}$. Fix an open set $A \subset \subset \Omega$ and let us prove that (17) holds for any ball $B_{r}\left(x_{0}\right) \subset A$ (with a constant $C_{0}$ not depending on $A$ ).

To this aim let $\psi \in C_{0}^{\infty}\left(\mathbb{R}^{n}\right)$ be a cut-off function such that $0 \leq \psi(x) \leq 1$ for all $x$, $\operatorname{supp} \psi \subset(-3,3)^{n}$ and such that $\psi(x) \equiv 1$ if $x \in[-1,1]^{n}$. For any $h \in \mathbb{N}$ we denote by $Q_{i, h}\left(x_{i, h}\right)$ the standard covering of $\mathbb{R}^{n}$ with closed cubes, centered at $x_{i, h}$, with sides parallel to the coordinates axes, side length equal to $2 / h$ and having pairwise disjoint interiors. Then, for any $i, h$, we set $\psi_{i, h}(x):=\psi\left(h\left(x-x_{i, h}\right)\right)$ and

$$
\sigma_{h}(x):=\sum_{i=1}^{\infty} \psi_{i, h}(x), \quad \varphi_{i, h}(x):=\frac{\psi_{i, h}(x)}{\sigma_{h}(x)}
$$

Finally, for all $h$ such that $12 \sqrt{n} / h<\operatorname{dist}(A ; \partial \Omega)$, and for every $x \in A, \xi \in \mathbb{R}^{n}$, we set

$$
f_{h}(x, \xi):=\sum_{i=1}^{\infty} \varphi_{i, h}(x) f\left(x_{i, h}, \xi\right) .
$$

Notice that the above sum is actually finite (indeed it consists of at most $3^{n}$ terms), and that each function $f_{h}$ is of the type considered in Step 1. Moreover, we claim that the functions $f_{h}$ satisfy the assumptions of 
Lemma 2.8 with suitable constants $L, \varepsilon_{0}, R, \nu$ not depending on $h$. The verification of our claim in the case of assumptions $(14,15)$ (or equivalently $(19))$ and (18) follows easily by the corresponding assumptions on $f$. We limit ourselves to show that for any $h$

$$
\left|D_{x \xi} f_{h}(x, \xi)\right| \leq c L\left(1+|\xi|^{p-1}\right), \quad \forall(x, \xi) \in A \times\left(\mathbb{R}^{n} \backslash \bar{B}_{R}\right)
$$

where $L$ is the constant appearing in (16) (relative to $f$ ) and $c$ is a constant depending only on $n, \psi$, but not on $h$. Let us fix $x_{0} \in A$ and $\xi \in \mathbb{R}^{n} \backslash \bar{B}_{R}$. By construction there exist at most $3^{n}$ cubes, $Q_{j_{1}, h}\left(x_{j_{1}, h}\right), \ldots, Q_{j_{3^{n}, h}}\left(x_{j_{3^{n}, h}}\right)$, such that for any $x$ in a suitable neighborhood $U$ of $x_{0}$

$$
f_{h}(x, \xi)=\sum_{l=1}^{3^{n}} \varphi_{j_{l}, h}(x) f\left(x_{j_{l}, h}, \xi\right), \quad \sum_{l=1}^{3^{n}} \varphi_{j_{l}, h}(x)=1
$$

Therefore for all $x \in U$ we have that

$$
D_{x \xi} f_{h}(x, \xi)=\sum_{l=1}^{3^{n}} D_{x} \varphi_{j_{l}, h}(x) D_{\xi} f\left(x_{j_{l}, h}, \xi\right)=\sum_{l=2}^{3^{n}} D_{x} \varphi_{j_{l}, h}(x)\left[D_{\xi} f\left(x_{j_{l}, h}, \xi\right)-D_{\xi} f\left(x_{j_{1}, h}, \xi\right)\right] .
$$

In view of assumption (16), we have that for all $l$,

$$
\left|D_{\xi} f\left(x_{j_{l}, h}, \xi\right)-D_{\xi} f\left(x_{j_{1}, h}, \xi\right)\right| \leq \frac{c(n) L}{h}\left(1+|\xi|^{p-1}\right) .
$$

On the other hand, for any $j$, there exists a set of indices $I_{j}$ containing $j$, with $\#\left(I_{j}\right)=3^{n}$, such that, for all $x \in \mathbb{R}^{n}$,

$$
D_{x} \varphi_{j, h}(x)=\frac{D_{x} \psi_{j, h}(x)}{\sigma_{h}(x)}-\frac{\psi_{j, h}(x)}{\sigma_{h}^{2}(x)} \sum_{k \in I_{j}} D_{x} \psi_{k, h}(x)
$$

Therefore, since by construction $\sigma_{h}(x) \geq 1$ for all $x$, we have that, for all $x \in \mathbb{R}^{n}$ and any $j$,

$$
\left|D_{x} \varphi_{j, h}(x)\right| \leq\left(3^{n}+1\right) h\left(\left\|D_{x} \psi\right\|_{\infty}\right) .
$$

In view of the above estimates and from (26), we may conclude that for all $(x, \xi) \in A \times\left(\mathbb{R}^{n} \backslash \bar{B}_{R}\right)$ and for any $h$

$$
\left|D_{x \xi} f_{h}(x, \xi)\right| \leq c(n) L\left\|D_{x} \psi\right\|_{\infty} \cdot\left(1+|\xi|^{p-1}\right),
$$

and thus (25) follows. Finally, notice that $f_{h}(x, \xi) \rightarrow f(x, \xi)$ uniformly in $A \times K$ for every $K$ compact subset of $\mathbb{R}^{n}$. Hence the rest of the proof goes as in Step 1, since also in this case the function $\xi \rightarrow f(x, \xi)$ is strictly convex for all $x \in A$.

Step 3: Let $f$ satisfy the assumptions of Theorem 2.7. Fix an open set $A \subset \subset \Omega$, an infinitesimal sequence $\varepsilon_{h}$ of positive numbers and a positive symmetric mollifier $\rho$. For $h$ large enough we set for all $(x, \xi) \in A \times \mathbb{R}^{n}$

$$
f_{h}(x, \xi):=\int_{B \times B} \rho(y) \rho(\eta) f\left(x+\varepsilon_{h} y, \xi+\varepsilon_{h} \eta\right) \mathrm{d} y \mathrm{~d} \eta+\varepsilon_{h}\left(1+|\xi|^{p}\right),
$$

where $B$ is the unit open ball in $\mathbb{R}^{n}$. Notice that each function $f_{h}$ is of the type considered in Step 2 and that $f_{h}(x, \xi) \rightarrow f(x, \xi)$ uniformly on any set of the type $A \times K$, with $K \subset \mathbb{R}^{n}$ compact. Moreover, the functions $f_{h}$ satisfy the assumptions of Theorem 2.7 with the corresponding constants $L, R, \nu$ bounded from above and away from zero. 
As in Step 1, given a ball $B_{r}\left(x_{0}\right) \subset A$ we denote by $u_{h}$ the solution of the problem

$$
\min \left\{\int_{B_{r}\left(x_{0}\right)} f_{h}(x, D v) \mathrm{d} x: v \in u+W^{1, p}\left(B_{r}\left(x_{0}\right)\right)\right\}
$$

From the assumptions on $f$ and the construction of the functions $f_{h}$ it follows easily that the sequence $u_{h}$ is bounded in $W^{1, p}\left(B_{r}\left(x_{0}\right)\right)$. Moreover, by Step 2, for all $\rho<r$ we have

$$
\sup _{B_{\rho}\left(x_{0}\right)}\left|D u_{h}\right|^{p} \leq C\left(1+f_{B_{r}\left(x_{0}\right)}\left|D u_{h}\right|^{p} \mathrm{~d} x\right)
$$

where the constant $C$ depends only on $L, p, R, \nu$ and $\rho$, but not on $h$. Hence we may assume that, up to a subsequence, $u_{h} \rightarrow u_{\infty}$ weakly* in $W^{1, \infty}\left(B_{\rho}\left(x_{0}\right)\right)$ for any $\rho<r$. As in Step 1 we have again that also $u_{\infty}$ is a minimizer of $F$ in $B_{r}\left(x_{0}\right)$. However, in the present case the functional $F$ is not necessarily strictly convex, hence we may not conclude as before that $u_{\infty}=u$ in $B_{r}\left(x_{0}\right)$.

Set $E_{0}:=\left\{x \in B_{r}\left(x_{0}\right):\left|D u_{\infty}(x)+D u(x)\right|>2 R_{0}\right\}$, where $R_{0}$ is the constant provided by Lemma 2.5. If $E_{0}$ has positive measure, then from the convexity of $f(x, \cdot)$ and Remark 2.6 we have, setting $\tilde{u}:=\left(u+u_{\infty}\right) / 2$,

$$
\int_{B_{\rho}\left(x_{0}\right) \backslash E_{0}} f(x, D \tilde{u}) \mathrm{d} x \leq \frac{1}{2} \int_{B_{\rho}\left(x_{0}\right) \backslash E_{0}} f(x, D u) \mathrm{d} x+\frac{1}{2} \int_{B_{\rho}\left(x_{0}\right) \backslash E_{0}} f\left(x, D u_{\infty}\right) \mathrm{d} x .
$$

Also, applying twice (8) in Lemma 2.5, first with $\xi:=D \tilde{u}$ and $\eta:=D u$, and then with $\xi:=D \tilde{u}$ and $\eta:=D u_{\infty}$, adding up these two inequalities yields

$$
\int_{B_{\rho}\left(x_{0}\right) \cap E_{0}} f(x, D \tilde{u}) \mathrm{d} x<\frac{1}{2} \int_{B_{\rho}\left(x_{0}\right) \cap E_{0}} f(x, D u) \mathrm{d} x+\frac{1}{2} \int_{B_{\rho}\left(x_{0}\right) \cap E_{0}} f\left(x, D u_{\infty}\right) \mathrm{d} x .
$$

Adding these two inequalities we get a contradiction with the minimality of $u$ and $u_{\infty}$. Therefore $E_{0}$ has zero measure. Applying Step 2 to the functions $u_{h}$, (8) to the functions $f_{h}$, and using the minimality of $u_{h}$, we deduce that

$$
\begin{aligned}
\sup _{B_{r / 2}\left(x_{0}\right)}\left|D u_{\infty}\right|^{p} & \leq \liminf _{h \rightarrow+\infty}\left(\sup _{B_{r / 2}\left(x_{0}\right)}\left|D u_{h}\right|^{p}\right) \\
& \leq \tilde{C} \liminf _{h \rightarrow+\infty}\left(1+f_{B_{r}\left(x_{0}\right)}\left|D u_{h}\right|^{p} \mathrm{~d} x\right) \leq c \liminf _{h \rightarrow+\infty}\left(1+f_{B_{r}\left(x_{0}\right)} f_{h}\left(x, D u_{h}\right) \mathrm{d} x\right) \\
& \leq c \liminf _{h \rightarrow+\infty}\left(1+f_{B_{r}\left(x_{0}\right)} f_{h}\left(x, D u_{\infty}\right) \mathrm{d} x\right) \leq c\left(1+f_{B_{r}\left(x_{0}\right)}\left|D u_{\infty}\right|^{p} \mathrm{~d} x\right) .
\end{aligned}
$$

Then the result follows, since $\left|D u(x)+D u_{\infty}(x)\right| \leq 2 R_{0}$ for a.e. $x$.

\section{Attainment of Minima FOR NONCONVEX PROBLEMS}

Here we give an existence result for the variational problem

$$
\inf \left\{\int_{\Omega} f(x, D u(x)) \mathrm{d} x: u \in u_{0}+W_{0}^{1, p}(\Omega)\right\},
$$


where $\Omega$ is a bounded open set of $\mathbb{R}^{n}$ and $u_{0} \in W^{1, p}(\Omega), p>1$. Throughout this section we assume that $f: \Omega \times \mathbb{R}^{n} \rightarrow \mathbb{R}$ is a continuous function satisfying the growth condition

$$
c_{1}|\xi|^{p}-c_{2} \leq f(x, \xi) \leq L\left(1+|\xi|^{p}\right), \quad \forall(x, \xi) \in \Omega \times \mathbb{R}^{n},
$$

for some constants $c_{1}, c_{2}, L>0$, and is uniformly convex at infinity with respect to $\xi$, i.e., there exist $R, \nu>0$ such that, if the segment $\left[\xi_{1}, \xi_{2}\right]$ is contained in $\mathbb{R}^{n} \backslash \bar{B}_{R}$, then

$$
f\left(x, \frac{\xi_{1}+\xi_{2}}{2}\right) \leq \frac{1}{2} f\left(x, \xi_{1}\right)+\frac{1}{2} f\left(x, \xi_{2}\right)-\nu\left(1+\left|\xi_{1}\right|^{2}+\left|\xi_{2}\right|^{2}\right)^{\frac{p-2}{2}}\left|\xi_{1}-\xi_{2}\right|^{2}
$$

for every $x \in \Omega$ (see (6)). Notice that, if $0 \leq f(x, \xi) \leq L\left(1+|\xi|^{p}\right)$, then condition (29) implies the coercivity inequality in the left hand side of (28) (see Rem. 2.6). In addition, we assume that there exists the distributional derivative $D_{x \xi} f(x, \xi)$ and

$$
\left|D_{x \xi} f(x, \xi)\right| \leq L\left(1+|\xi|^{p-1}\right), \quad \forall x \in \Omega,|\xi|>R,
$$

provided $L$ in (28) is chosen to be sufficiently large (see (16)). Let us denote by $f^{* *}:=f^{* *}(x, \xi)$ the bipolar of $f$, that is the convex envelope of $f(x, \cdot)$ (i.e., the largest convex function in $\xi$ which is less than or equal to $f(x, \cdot)$ on $\left.\mathbb{R}^{n}\right)$. We assume that $f^{* *}$ is continuous; hence, for any $x \in \Omega$, the set

$$
A(x):=\left\{\xi \in \mathbb{R}^{n}: f(x, \xi)>f^{* *}(x, \xi)\right\}
$$

is open.

We shall prove the existence of a minimizer for the problem $(27)$ under the main assumption that $f^{* *}(x, \cdot)$ is affine on each connected component of $A(x)$. However, in order to present the argument of the proof in a simplified setting, we shall treat first the case where $f^{* *}(x, \cdot)$ is affine (with the same slope) on the whole set $A(x)$. We will refer to this situation as the model case. The proof of this case contains all the ideas and technical tools which are needed to treat the general situation in which $f^{* *}(x, \cdot)$ is affine (with possibly different slopes) on each connected component of $A(x)$.

\subsection{The model case}

As before $f: \Omega \times \mathbb{R}^{n} \rightarrow \mathbb{R}$ is a continuous function satisfying (28-30). We assume that $f^{* *}: \Omega \times \mathbb{R}^{n} \rightarrow \mathbb{R}$ is continuous and we denote by $A(x)$ the set defined in (31) and by $\Omega_{A}:=\{x \in \Omega: A(x) \neq \emptyset\}$. Since $f$ and $f^{* *}$ are continuous functions, $\Omega_{A}$ is open. Here we consider the case where $f^{* *}(x, \cdot)$ is affine in $A(x)$; more precisely, we assume that there exist a function $q \in C^{0}\left(\Omega_{A}\right)$ and a vector field $m \in C^{1}\left(\Omega_{A} ; \mathbb{R}^{n}\right)$ such that

$$
f^{* *}(x, \xi)=q(x)+\langle m(x), \xi\rangle, \quad \forall x \in \Omega_{A}, \xi \in A(x) .
$$

We also assume that the boundary of the set where the divergence of $m$ is equal to zero is negligible, i.e.,

$$
\text { meas }\left(\Omega \cap \partial\left\{x \in \Omega_{A}: \operatorname{div} m(x)=0\right\}\right)=0 .
$$

Finally, for every $x \in \Omega_{A}$, we set

$$
E(x):=\left\{\xi \in \mathbb{R}^{n}: f^{* *}(x, \xi)=q(x)+\langle m(x), \xi\rangle\right\} ;
$$

note that, by the growth conditions in (28) and by the assumption that $f$ is uniformly convex at infinity with respect to $\xi$, then the set $E(x)$ is bounded uniformly for $x \in \Omega_{A}$ (see also Lem. 2.5). We assume 
there exists an increasing function $\omega:[0,+\infty) \rightarrow[0,+\infty)$, with $\omega(t)=0$ if and only if $t=0$, such that, if $x \in \Omega_{A}, \xi \in \partial E(x), \eta \in \mathbb{R}^{n} \backslash E(x)$, then

$$
f^{* *}\left(x, \frac{\xi+\eta}{2}\right) \leq \frac{1}{2} f^{* *}(x, \xi)+\frac{1}{2} f^{* *}(x, \eta)-\omega(|\xi-\eta|) .
$$

The main result of this section is the following existence theorem:

Theorem 3.1. Let $f, f^{* *}: \Omega \times \mathbb{R}^{n} \rightarrow \mathbb{R}$ be continuous functions ( $f$ not necessarily convex with respect to $\left.\xi \in \mathbb{R}^{n}\right)$. Under the above assumptions on $f$ and $f^{* *}$ ((28-30) and (32-34)), for any given boundary datum $u_{0} \in W^{1, p}(\Omega)$ the variational problem (27) attains its minimum. Moreover, any minimizers is of class $W_{\text {loc }}^{1, \infty}(\Omega)$.

The proof of Theorem 3.1 is obtained using the same method as in the work of Dacorogna-Marcellini [12,13], who considered integrands independent of $x$. Our proof however follows from some new lemmas. The first one concerns the relaxed variational problem

$$
\inf \left\{\int_{\Omega} f^{* *}(x, D u(x)) \mathrm{d} x: u \in u_{0}+W_{0}^{1, p}(\Omega)\right\} .
$$

Lemma 3.2. The minimum of the relaxed variational problem (35) is attained. Moreover, there exist a minimizer $v \in W_{\text {loc }}^{1, \infty}(\Omega) \cap\left(u_{0}+W_{0}^{1, p}(\Omega)\right)$ of (35) and an open set $\Omega^{\prime} \subset \Omega$ (possibly empty) such that

$$
\left\{\begin{array}{lll}
D v(x) \in A(x) & \text { a.e. } & x \in \Omega^{\prime} \\
D v(x) \notin A(x) & \text { a.e. } & x \in \Omega \backslash \Omega^{\prime}
\end{array}\right.
$$

and $\operatorname{div} m=0$ in $\Omega^{\prime}$.

Remark 3.3. Formally, if $D v(x) \in A(x)$ then by (32)

$$
f^{* *}(x, D v(x))=q(x)+\langle m(x), D v(x)\rangle \cdot
$$

Therefore, the Euler's equation for $v$ gives

$$
\sum_{s=1}^{n} \frac{\partial}{\partial x_{s}} f_{\xi_{s}}^{* *}(x, D v)=\operatorname{div} m(x)=0, \quad \text { a.e. } x \text { such that } D v(x) \in A(x) .
$$

Thus (36) would follow if we could define $\Omega^{\prime}:=\{D v(x) \in A(x)\}$. A striking feature of Lemma 3.2 is that the set $\Omega^{\prime}$ may be chosen to be open, and so Lemma 3.2 may be considered to be a strong form of Euler's first variation for the minimizers. The property (36) is a regularity result, and in fact it follows from the regularity results obtained in Section 2.

The proof of Lemma 3.2 follows the argument by Dacorogna-Marcellini [12] in Theorem 10.9. Previous arguments related to Lemma 3.2 are due to De Blasi-Pianigiani [14], Sychev [28], and Zagatti [29].

Proof of Lemma 3.2. As before we denote by $\Omega_{A}$ the open subset of $\Omega$ consisting of those points $x$ such that $A(x) \neq \emptyset$. We split $\Omega_{A}$ into three sets (possibly empty),

$$
\begin{gathered}
\Omega_{A}^{+}:=\left\{x \in \Omega_{A}: \operatorname{div} m(x)>0\right\}, \quad \Omega_{A}^{-}:=\left\{x \in \Omega_{A}: \operatorname{div} m(x)<0\right\} \\
\Omega_{A}^{0}:=\left\{x \in \Omega_{A}: \operatorname{div} m(x)=0\right\}
\end{gathered}
$$


Since $\operatorname{div} m$ is a continuous function, $\Omega_{A}^{+} \cup \Omega_{A}^{-} \cup \operatorname{int} \Omega_{A}^{0}$ is an open set and, by (33),

$$
\text { meas }\left(\Omega_{A} \backslash\left(\Omega_{A}^{+} \cup \Omega_{A}^{-} \cup \operatorname{int} \Omega_{A}^{0}\right)\right) \leq \operatorname{meas}\left(\Omega \cap \partial \Omega_{A}^{0}\right)=0 .
$$

By (28-30), Lemma 2.5, and Theorem 2.7 the relaxed variational problem (35) has a minimizer $u^{* *}$ in the Sobolev class $W_{\text {loc }}^{1, \infty}(\Omega) \cap\left(u_{0}+W^{1, p}(\Omega)\right)$. Thus, by Rademacher theorem (see, for example, Th. 2.2.1 of [30], or Th. 2.14 of [2]) $u^{* *}$ is classically differentiable for almost every $x \in \Omega$.

Let $x_{0}$ be a point of $\Omega_{A} \backslash \partial \Omega_{A}^{0}$ where $u^{* *}$ is differentiable. Then

$$
u^{* *}(x)=u^{* *}\left(x_{0}\right)+\left\langle D u^{* *}\left(x_{0}\right), x-x_{0}\right\rangle+o\left(\left|x-x_{0}\right|\right), x \in \Omega .
$$

Also, assume that

$$
D u^{* *}\left(x_{0}\right) \in A\left(x_{0}\right)=\left\{\xi \in \mathbb{R}^{n}: f\left(x_{0}, \xi\right)>f^{* *}\left(x_{0}, \xi\right)\right\} .
$$

Since $\Omega_{A}$ and $A(x)$ are open sets, there exists $\gamma \in(0,1)$ (depending on $u^{* *}$ and $x_{0}$ ) such that

$$
x \in \Omega_{A}, \quad \xi \in A(x),
$$

for all $(x, \xi) \in \Omega \times \mathbb{R}^{n}$ such that

$$
\left|x-x_{0}\right| \leq \gamma,\left|\xi-D u^{* *}\left(x_{0}\right)\right| \leq 2 \gamma .
$$

Recall that $x_{0} \in \Omega_{A} \backslash \partial \Omega_{A}^{0}$; thus we can also assume that $\gamma$ is sufficiently small so that

$$
\left\{\begin{array}{l}
x_{0} \in \Omega_{A}^{ \pm},\left|x-x_{0}\right| \leq \gamma \quad \Longrightarrow \quad x \in \Omega_{A}^{ \pm} \\
x_{0} \in \operatorname{int} \Omega_{A}^{0},\left|x-x_{0}\right| \leq \gamma \quad \Longrightarrow \quad x \in \operatorname{int} \Omega_{A}^{0} .
\end{array}\right.
$$

Choose $\delta \in(0, \gamma]$ (depending on $\left.x_{0}\right)$ sufficiently small such that

$$
\frac{\left|o\left(\left|x-x_{0}\right|\right)\right|}{\left|x-x_{0}\right|} \leq \gamma, \quad \forall x \in \bar{B}_{\delta}\left(x_{0}\right), x \neq x_{0},
$$

and

$$
\delta \leq \frac{\gamma}{2\left|D u^{* *}\left(x_{0}\right)\right|+4 \gamma}, \quad \bar{B}_{\delta}\left(x_{0}\right) \subset \Omega .
$$

By (42) and by the definition of $\Omega_{A}^{+}, \Omega_{A}^{-}, \Omega_{A}^{0}$ in $(37,38)$, we have

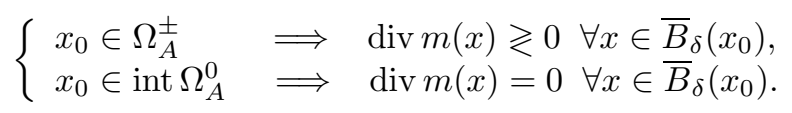

For every $r \in(0, \delta]$, let us define in $\Omega$ the function $v_{x_{0}}^{r}$ by

$$
v_{x_{0}}^{r}(x):=u^{* *}\left(x_{0}\right)+\left\langle D u^{* *}\left(x_{0}\right), x-x_{0}\right\rangle \pm \gamma \cdot\left(r-2\left|x-x_{0}\right|\right), \quad x \in \Omega,
$$

the sign + being chosen if $x_{0} \in \Omega_{A}^{+}$, while the sign - is selected if $x_{0} \in \Omega_{A}^{-}$. If $x_{0} \in \operatorname{int} \Omega_{A}^{0}$ then any sign in the definition of $v_{x_{0}}^{r}(x)$ is a good choice; in order to fix the ideas, we choose the sign + if $x_{0} \in \operatorname{int} \Omega_{A}^{0}$. Since $|D| x||=1$ for every $x \in \mathbb{R}^{n} \backslash\{0\}$, we obtain

$$
\left|D v_{x_{0}}^{r}(x)-D u^{* *}\left(x_{0}\right)\right|=2 \gamma \cdot|\mp D| x-x_{0}||=2 \gamma \quad \text { a.e. } x \in \Omega,
$$


and thus by $(40,41)$,

$$
D v_{x_{0}}^{r}(x) \in A(x), \quad \text { a.e. } x \in \bar{B}_{\delta}\left(x_{0}\right), \quad \forall r \in(0, \delta]
$$

If $x_{0} \in \Omega_{A}^{+} \cup \operatorname{int} \Omega_{A}^{0}$ we set

$$
G\left(x_{0}, r\right):=\left\{x \in \bar{B}_{\delta}\left(x_{0}\right) \subset \Omega: v_{x_{0}}^{r}(x) \geq u^{* *}(x)\right\}
$$

and if $x_{0} \in \Omega_{A}^{-}$we define

$$
G\left(x_{0}, r\right):=\left\{x \in \bar{B}_{\delta}\left(x_{0}\right) \subset \Omega: v_{x_{0}}^{r}(x) \leq u^{* *}(x)\right\}
$$

We claim that $G\left(x_{0}, r\right)$ is a closed set satisfying

$$
\bar{B}_{r / 3}\left(x_{0}\right) \subset G\left(x_{0}, r\right) \subset \bar{B}_{r}\left(x_{0}\right) .
$$

Let us verify (49) when $x_{0} \in \Omega_{A}^{+} \cup$ int $\Omega_{A}^{0}$. If $x \in \bar{B}_{\delta}\left(x_{0}\right)$ but $x \notin \bar{B}_{r}\left(x_{0}\right)$ (that is $r<\left|x-x_{0}\right| \leq \delta$ ) then by (39) and (43) we get

$$
\begin{aligned}
v_{x_{0}}^{r}(x)-u^{* *}(x)=\gamma \cdot\left(r-2\left|x-x_{0}\right|\right)-o\left(\left|x-x_{0}\right|\right) & <-\gamma\left|x-x_{0}\right|-o\left(\left|x-x_{0}\right|\right) \\
& =-\left|x-x_{0}\right| \cdot\left(\gamma+\frac{o\left(\left|x-x_{0}\right|\right)}{\left|x-x_{0}\right|}\right) \leq 0
\end{aligned}
$$

Thus $v_{x_{0}}^{r}(x)-u^{* *}(x)<0$ and $x \notin G\left(x_{0}, r\right)$. On the other hand if $x \in \bar{B}_{r / 3}\left(x_{0}\right)$, then $r / 3 \geq\left|x-x_{0}\right|$, and again by $(39,43)$, we obtain

$$
\begin{aligned}
v_{x_{0}}^{r}(x)-u^{* *}(x)=\gamma \cdot\left(r-2\left|x-x_{0}\right|\right)-o\left(\left|x-x_{0}\right|\right) & \geq \gamma\left|x-x_{0}\right|-o\left(\left|x-x_{0}\right|\right) \\
& =\left|x-x_{0}\right|\left(\gamma-\frac{o\left(\left|x-x_{0}\right|\right)}{\left|x-x_{0}\right|}\right) \geq 0
\end{aligned}
$$

and $x \in G\left(x_{0}, r\right)$. Thus (49) is proved.

By (47) and the continuity of $u^{* *}$ and $v_{x_{0}}^{r}$ we have

$$
\partial G\left(x_{0}, r\right) \subset\left\{x \in \bar{B}_{\delta}\left(x_{0}\right): v_{x_{0}}^{r}(x)=u^{* *}(x)\right\}
$$

thus $\partial G\left(x_{0}, r\right)$ and $\partial G\left(x_{0}, r^{\prime}\right)$ are disjoint for $r \neq r^{\prime}$ and we conclude that only countably many of these boundary sets can have positive measure. Therefore we can always choose a sequence $r_{h}$ of real numbers such that

$$
\left\{\begin{array}{l}
r_{h} \rightarrow 0 \text { as } h \rightarrow+\infty \\
0<r_{h} \leq \delta, \forall h \in \mathbb{N} \\
\text { meas }\left(\partial G\left(x_{0}, r_{h}\right)\right)=0, \quad \forall h \in \mathbb{N}
\end{array}\right.
$$

Let us consider the measurable subset of $\Omega$

$$
M:=\left\{x_{0} \in \Omega_{A}^{+} \cup \Omega_{A}^{-} \cup \operatorname{int} \Omega_{A}^{0}: u^{* *} \text { differentiable at } x_{0}, \quad D u^{* *}\left(x_{0}\right) \in A\left(x_{0}\right)\right\}
$$

and consider the family of open sets

$$
\mathcal{G}:=\left\{\operatorname{int} G\left(x, r_{h}\right): x \in M, r_{h} \text { as in }(50)\right\}
$$


Since $\mathcal{G}$ is a fine covering of $M$ and each set $G(x, r) \in \mathcal{G}$ satisfies (49), by Vitali's covering theorem (see, for example, Chap. 10 of [12]), there exists in $\mathcal{G}$ a (at most) countable subcollection $\mathcal{G}^{\prime}$ of sets with disjoint closures such that the open set

$$
\Omega^{\prime}=\bigcup_{\operatorname{int} G\left(x_{k}, r_{k}\right) \in \mathcal{G}^{\prime}} \operatorname{int} G\left(x_{k}, r_{k}\right)
$$

covers $M$ up to a set of zero measure, i.e. meas $\left(M \backslash \Omega^{\prime}\right)=0$. Let us define the function $v$ in $\Omega$ by

$$
v(x):= \begin{cases}u^{* *}(x) & \text { if } x \in \Omega \backslash \Omega^{\prime} \\ v_{x_{k}}^{r_{k}}(x) & \text { if } x \in G\left(x_{k}, r_{k}\right)\end{cases}
$$

and introduce the functions

$$
u_{h}(x):=\left\{\begin{array}{l}
u^{* *}(x) \text { if } x \in \Omega \backslash \cup_{k=1}^{h} G\left(x_{k}, r_{k}\right) \\
v_{x_{k}}^{r_{k}}(x) \text { if } x \in G\left(x_{k}, r_{k}\right), \text { for some } 1 \leq k \leq h
\end{array}\right.
$$

By (47) and (48) the functions $u_{h}$ belong to $u^{* *}+W_{0}^{1, p}(\Omega) \cap W_{\text {loc }}^{1, \infty}(\Omega)$ for all $h$, since $u_{h}$ is locally maximum or minimum of two $W^{1, p}(\Omega) \cap W_{\text {loc }}^{1, \infty}(\Omega)$ functions. Moreover, we claim that each $u_{h}$ is a minimizer of (35). To this aim, notice that by $(45,47,48)$, we have for all $h$

$$
\operatorname{div} m(x)\left(u_{h}(x)-u^{* *}(x)\right) \geq 0 \quad \text { a.e. } x \in \cup_{k=1}^{h} G\left(x_{k}, r_{k}\right) .
$$

Moreover, by (46)

$$
D u_{h}(x) \in A(x), \quad \text { a.e. } x \in \cup_{k=1}^{h} G\left(x_{k}, r_{k}\right) .
$$

and $D u_{h}(x)=D u^{* *}(x)$ a.e. in $\Omega \backslash \cup_{k=1}^{h} G\left(x_{k}, r_{k}\right)$. By the convexity inequality $f^{* *}(x, \xi) \geq q(x)+\langle m(x), \xi\rangle$, valid for every $x \in \Omega_{A}$ and every $\xi \in \mathbb{R}^{n}$, we have

$$
\begin{aligned}
\inf \left\{\int_{\Omega} f^{* *}(x, D u(x)) \mathrm{d} x: u \in u_{0}+W_{0}^{1, p}(\Omega)\right\}= & \int_{\Omega \backslash \cup_{k=1}^{h} G\left(x_{k}, r_{k}\right)} f^{* *}\left(x, D u^{* *}(x)\right) \mathrm{d} x \\
& +\int_{\cup_{k=1}^{h} G\left(x_{k}, r_{k}\right)} f^{* *}\left(x, D u^{* *}(x)\right) \mathrm{d} x \\
\geq & \int_{\Omega \backslash \cup_{k=1}^{h} G\left(x_{k}, r_{k}\right)} f^{* *}\left(x, D u^{* *}(x)\right) \mathrm{d} x \\
& +\int_{\cup_{k=1}^{h} G\left(x_{k}, r_{k}\right)}\left\{q(x)+\left\langle m(x), D u^{* *}(x)\right\rangle\right\} \mathrm{d} x,
\end{aligned}
$$

where we have used the fact that $\cup_{k=1}^{h} G\left(x_{k}, r_{k}\right) \subset \Omega_{A}$ (see $(40,41)$ and (49)).

Since $u_{h} \in u^{* *}+W_{0}^{1, p}\left(\operatorname{int} G\left(x_{k}, r_{k}\right)\right)$ for all $1 \leq k \leq h$, using (53) we obtain

$$
\int_{G\left(x_{k}, r_{k}\right)}\left\{\left\langle m(x), D u^{* *}(x)\right\rangle-\left\langle m(x), D u_{h}(x)\right\rangle\right\} \mathrm{d} x=-\int_{G\left(x_{k}, r_{k}\right)} \operatorname{div} m(x)\left(u^{* *}(x)-u_{h}(x)\right) \mathrm{d} x \geq 0 .
$$


Therefore we may conclude, from the inequality above and by (54), that

$$
\begin{aligned}
\inf \left\{\int_{\Omega} f^{* *}(x, D u(x)) \mathrm{d} x: u \in u_{0}+W_{0}^{1, p}(\Omega)\right\} \geq & \int_{\Omega \backslash \cup_{k=1}^{h} G\left(x_{k}, r_{k}\right)} f^{* *}\left(x, D u^{* *}(x)\right) \mathrm{d} x \\
& +\int_{\cup_{k=1}^{h} G\left(x_{k}, r_{k}\right)}\left\{q(x)+\left\langle m(x), D u_{h}(x)\right\rangle\right\} \mathrm{d} x \\
= & \int_{\Omega} f^{* *}\left(x, D u_{h}(x)\right) \mathrm{d} x .
\end{aligned}
$$

This proves the minimality of each $u_{h}$. By $(28)$ the sequence $u_{h}$ is bounded in $W^{1, p}(\Omega)$ and since $u_{h}(x)$ converges to $v(x)$ a.e. in $\Omega$, it follows that $u_{h}$ converges weakly to $v$ in $W^{1, p}(\Omega)$. In particular, $v \in u_{0}+W_{0}^{1, p}(\Omega)$ and, by lower semicontinuity, $v$ is also a minimizer of (35). Theorem 2.7 now yields $v \in W_{\text {loc }}^{1, \infty}(\Omega)$. By (46) we have

$$
D v(x) \in A(x) \text {, a.e. } x \in \Omega^{\prime} .
$$

On the other hand, since $v=u^{* *}$ in $\Omega \backslash \Omega^{\prime}$, there exists a negligible set $E_{0}$ such that, for all $x \in\left(\Omega \backslash \Omega^{\prime}\right) \backslash E_{0}$, $v$ and $u^{* *}$ are differentiable at $x$ and $D v(x)=D u^{* *}(x)$. Thus, if $x \notin E_{0} \cup\left(M \backslash \Omega^{\prime}\right) \cup\left(\Omega \cap \partial \Omega_{A}^{0}\right)$, which is a set of measure zero, and if $x \notin \Omega \backslash \Omega^{\prime}$, then we have

$$
D v(x) \notin A(x) .
$$

Finally, let us prove that $\operatorname{div} m=0$ in $\Omega^{\prime}$. To this end notice that, since

$$
f^{* *}(x, \xi)=q(x)+\langle m(x), \xi\rangle \quad \text { whenever } x \in \Omega_{A} \text { and } \xi \in A(x),
$$

the function $\xi \rightarrow f^{* *}(x, \xi)$ is differentiable in $A(x)$ and $D_{\xi} f^{* *}(x, \xi)=m(x)$ for every $x \in \Omega^{\prime}, \xi \in A(x)$. Therefore the standard argument used to derive the Euler equation of an integral functional still applies in the open set $\Omega^{\prime}$ and the Euler's equation in weak form gives

$$
\int_{\Omega^{\prime}} \operatorname{div} m(x) \varphi(x) \mathrm{d} x=0, \quad \forall \varphi \in C_{0}^{1}\left(\Omega^{\prime}\right),
$$

which proves that $\operatorname{div} m=0$ in $\Omega^{\prime}$.

The second lemma that we consider in this section uses the notion of Kuratowski convergence, or convergence in the Hausdorff metric of a sequence of compact sets of $\mathbb{R}^{n}$. We recall that a sequence $E_{h}$ of compact sets of $\mathbb{R}^{n}$ converges, as $h \rightarrow+\infty$, in the sense of Kuratowski (or in the Hausdorff metric) to a compact set $E \subset \mathbb{R}^{n}$ if, for every $\varepsilon>0$, there exists $h_{0}$ such that

$$
E_{h} \subset I_{\varepsilon}(E), \quad E \subset I_{\varepsilon}\left(E_{h}\right) \quad \forall h>h_{0},
$$

where $I_{\varepsilon}(\cdot)$ denotes the neighborhood of radius $\varepsilon$ of the set under consideration. We recall that, if the sequence $E_{h}$ is bounded in $\mathbb{R}^{n}$ uniformly with respect to $h \in \mathbb{N}$, then $E_{h} \rightarrow E$ in the sense of Kuratowski if and only if the following two properties hold:

(i) for every $\xi \in E$ there exists a sequence $\xi_{h}$, with $\xi_{h} \in E_{h}$ for every $h \in \mathbb{N}$, such that $\xi_{h}$ converges to $\xi$ as $h \rightarrow+\infty$;

(ii) if a sequence $\xi_{h}$, with $\xi_{h} \in E_{h}$ for every $h \in \mathbb{N}$, admits a subsequence $\xi_{h_{k}}$ converging to a point $\xi$, then $\xi \in E$. 
In the sequel we need some properties of the distance function to a convex set. For the statements and the proofs, we refer the reader to the Appendix.

Let $E(x) \neq \emptyset$ be a compact subset of $\mathbb{R}^{n}$ for every value of a parameter $x$ in an open set $\Omega_{A} \subset \mathbb{R}^{n}$. We say that the set function $E(x)$ is continuous in the sense of Kuratowski if $E\left(x_{k}\right)$ converges in the sense of Kuratowski to $E(x)$ for every $x, x_{k} \in \Omega_{A}$ such that $x_{k} \rightarrow x$.

Lemma 3.4. Let $f: \Omega_{A} \times \mathbb{R}^{n} \rightarrow \mathbb{R}$ be a continuous function such that the bipolar $f^{* *}$ is also continuous and satisfies (32) and (34). Let $E(x) \subset \mathbb{R}^{n}$ be defined by

$$
E(x):=\left\{\xi \in \mathbb{R}^{n}: f^{* *}(x, \xi)-[q(x)+\langle m(x), \xi\rangle]=0\right\} .
$$

Then the map $x \in \Omega_{A} \mapsto E(x)$ is continuous in the sense of Kuratowski.

Proof. Let $x_{h}$ be a sequence in $\Omega_{A}$ converging to $x \in \Omega_{A}$. We claim that, if $\xi_{h_{k}} \in E\left(x_{h_{k}}\right)$ and $\xi_{h_{k}} \rightarrow \xi$, then $\xi \in E(x)$. In fact, since

$$
f^{* *}\left(x_{h_{k}}, \xi_{h_{k}}\right)=q\left(x_{h_{k}}\right)+\left\langle m\left(x_{h_{k}}\right), \xi_{h_{k}}\right\rangle,
$$

the continuity of $f^{* *}, m$, and $q$ yields $\xi \in E(x)$.

Let us now show that, given $\xi \in E(x)$, there exist $\xi_{h} \in E\left(x_{h}\right)$ such that $\xi_{h} \rightarrow \xi$. Indeed, if this is not true, then we can find $\varepsilon>0$ and a sequence $h_{k}$ such that $\operatorname{dist}\left(\xi ; E\left(x_{h_{k}}\right)\right)>\varepsilon$ for all $k \in \mathbb{N}$. For every $k \in \mathbb{N}$ choose $\xi_{h_{k}} \in \partial E\left(x_{h_{k}}\right)$ such that $\left|\xi-\xi_{h_{k}}\right|=\operatorname{dist}\left(\xi ; E\left(x_{h_{k}}\right)\right)>\varepsilon$. Extracting, if necessary, a further subsequence, we may assume that $\xi_{h_{k}} \rightarrow \eta$, and $\eta \in E(x)$ by the first part of the proof. Since $\xi \notin E\left(x_{h_{k}}\right)$ and $\xi_{h_{k}} \in \partial E\left(x_{h_{k}}\right)$, from (34) we get, for all $k \in \mathbb{N}$,

$$
f^{* *}\left(x_{h_{k}}, \frac{\xi+\xi_{h_{k}}}{2}\right) \leq \frac{1}{2} f^{* *}\left(x_{h_{k}}, \xi\right)+\frac{1}{2} f^{* *}\left(x_{h_{k}}, \xi_{h_{k}}\right)-\omega\left(\left|\xi-\xi_{h_{k}}\right|\right) .
$$

Passing to the limit as $k \rightarrow+\infty$, we get

$$
f^{* *}\left(x, \frac{\xi+\eta}{2}\right) \leq \frac{1}{2} f^{* *}(x, \xi)+\frac{1}{2} f^{* *}(x, \eta)-\omega(\varepsilon) .
$$

Since $f^{* *}(x, \cdot)$ is affine on $E(x)$ and $\xi, \eta \in E(x)$, the inequality above implies $\omega(\varepsilon) \leq 0$, which gives a contradiction since $\omega(t)>0$ for all $t>0$.

Lemma 3.5. Under the same assumptions of Lemma 3.4 the function $F: \Omega_{A} \times \mathbb{R}^{n} \rightarrow \mathbb{R}$, defined by

$$
F(x, \xi)=\left\{\begin{array}{l}
\operatorname{dist}(\xi ; \partial E(x)) \quad \text { if } \xi \in \mathbb{R}^{n} \backslash E(x), \\
-\operatorname{dist}(\xi ; \partial E(x)) \quad \text { if } \xi \in E(x),
\end{array}\right.
$$

is continuous in $\Omega_{A} \times \mathbb{R}^{n}$ and convex with respect to $\xi \in \mathbb{R}^{n}$.

Proof. The convexity of $F(x, \cdot)$ follows from Lemma 4.3 and the convexity of $E(x)$. The continuity of $F$ with respect to $x \in \Omega_{A}$ is a direct consequence of Lemma 4.2, asserting the continuity of the distance function with respect to the Kuratowski convergence, and of Lemma 3.4, yielding the continuity of the set function $E(x)$ with respect to $x$. The continuity of $F$ with respect to $(x, \xi)$ then follows from the fact that for all $x \in \Omega_{A}, \xi, \eta \in \mathbb{R}^{n}$ we have that $|F(x, \xi)-F(x, \eta)| \leq|\xi-\eta|$.

We are now ready to give the proof of Theorem 3.1. 
Proof of Theorem 3.1. By Lemma 3.2 the minimum of the relaxed variational problem (35) is attained and there exist a minimizer $v$ and an open set $\Omega^{\prime} \subset \Omega$ (possibly empty) such that

$$
\begin{cases}D v(x) \in A(x) & \text { a.e. } x \in \Omega^{\prime} \\ D v(x) \notin A(x) & \text { a.e. } x \in \Omega \backslash \Omega^{\prime}\end{cases}
$$

with $\operatorname{div} m=0$ in $\Omega^{\prime}$.

If $\Omega^{\prime}$ is an empty set then $D v(x) \notin A(x)$ a.e. $x \in \Omega$, and so $f^{* *}(x, D v(x))=f(x, D v(x))$ a.e. $x \in \Omega$, and $v$ is also a minimizer for the original problem $(27)$.

Otherwise, recalling (51), we consider for all $k$ the Dirichlet problem for the implicit differential equation

$$
\left\{\begin{array}{l}
F(x, D u(x))=0, \quad \text { a.e. } x \in \operatorname{int} G\left(x_{k}, r_{k}\right) \\
u(x)=v(x), \quad x \in \partial G\left(x_{k}, r_{k}\right)
\end{array}\right.
$$

where $F: \Omega_{A} \times \mathbb{R}^{n} \rightarrow \mathbb{R}$ is the signed distance function defined in Lemma 3.5. Notice that $v$ is Lipschitz continuous on $G\left(x_{k}, r_{k}\right)$, and since $A(x) \subset E(x)$ we deduce that

$$
F(x, D v(x)) \leq 0 \quad \text { a.e. } x \in \operatorname{int} G\left(x_{k}, r_{k}\right) .
$$

Therefore, we may apply Theorem 2.3 by Dacorogna-Marcellini [12] (note that (56) is exactly the compatibility condition (2.6) required in Th. 2.3 in [12]) to obtain the existence of a function $v_{k} \in W^{1, \infty}\left(\operatorname{int} G\left(x_{k}, r_{k}\right)\right)$ satisfying (55). Notice that $F(x, \xi)=0$ if and only if $\xi \in \partial E(x)$, and thus $D v_{k}(x) \in \partial E(x) \subset \mathbb{R}^{n} \backslash A(x)$ almost everywhere in int $G\left(x_{k}, r_{k}\right)$. Therefore

$$
f^{* *}\left(x, D v_{k}(x)\right)=f\left(x, D v_{k}(x)\right) \text { a.e. } x \in \operatorname{int} G\left(x_{k}, r_{k}\right) .
$$

Let us now prove that the functions

$$
w_{h}(x):= \begin{cases}v_{k}(x) & \text { if } x \in G\left(x_{k}, r_{k}\right), 1 \leq k \leq h, \\ v(x) & \text { if } x \in \Omega \backslash \cup_{k=1}^{h} G\left(x_{k}, r_{k}\right),\end{cases}
$$

are all minimizers in the class $u_{0}+W_{0}^{1, p}(\Omega)$ of the integral in (35). In fact, by the affinity assumption (32), for any $h$ we get

$$
\begin{aligned}
\int_{\Omega} f^{* *}(x, D v(x)) \mathrm{d} x-\int_{\Omega} f^{* *}\left(x, D w_{h}(x)\right) \mathrm{d} x & =\sum_{k=1}^{h} \int_{G\left(x_{k}, r_{k}\right)}\left\{f^{* *}(x, D v(x))-f^{* *}\left(x, D v_{k}(x)\right)\right\} \mathrm{d} x \\
& =\sum_{k=1}^{h} \int_{G\left(x_{k}, r_{k}\right)}\left\langle m(x), D v(x)-D v_{k}(x)\right\rangle \mathrm{d} x=0
\end{aligned}
$$

where we have used the fact that $v=v_{k}$ on $\partial G\left(x_{k}, r_{k}\right)$, together with the property that $\operatorname{div} m=0$ in $\Omega^{\prime}$. The minimality of the function $w_{h}$ follows immediately.

The proof will be over once we show that $w_{h}$ converges strongly in $W^{1, p}(\Omega)$ to a function $w$. Indeed, the limiting function $w$ will then be the required minimizer because by $(28,58)$, and $(57)$,

$$
\begin{aligned}
\int_{\Omega} f(x, D w(x)) \mathrm{d} x= & \lim _{h \rightarrow+\infty} \int_{\Omega} f\left(x, D w_{h}(x)\right) \mathrm{d} x=\int_{\Omega} f^{* *}(x, D v(x)) \mathrm{d} x \\
& +\lim _{h \rightarrow+\infty}\left[\int_{\Omega} f\left(x, D w_{h}(x)\right) \mathrm{d} x-\int_{\Omega} f^{* *}\left(x, D w_{h}(x)\right) \mathrm{d} x\right]=\int_{\Omega} f^{* *}(x, D v(x)) \mathrm{d} x \\
& \left.+\lim _{h \rightarrow+\infty} \int_{\Omega^{\prime} \backslash\left(\cup_{k \leq h} G\left(x_{k}, r_{k}\right)\right)}(x, D v(x))-f^{* *}(x, D v(x))\right] \mathrm{d} x=\int_{\Omega} f^{* *}(x, D v(x)) \mathrm{d} x
\end{aligned}
$$


where we have used the fact that $f(x, D v(x))=f^{* *}(x, D v(x))$ for a.e. $x \notin \Omega^{\prime}$. In order to prove that $w_{h}$ converges in $W^{1, p}(\Omega)$, it suffices to show that $D w_{h}$ is a Cauchy sequence in $L^{p}$. Since $v_{k}$ minimizes the integral

$$
\int_{G\left(x_{k}, r_{k}\right)} f^{* *}(x, D u(x)) \mathrm{d} x
$$

in the class $v+W_{0}^{1, p}\left(\operatorname{int} G\left(x_{k}, r_{k}\right)\right)$, by (28) there exists a constant $c$ such that for all $k$

$$
\int_{G\left(x_{k}, r_{k}\right)}\left|D v_{k}\right|^{p} \mathrm{~d} x \leq c \int_{G\left(x_{k}, r_{k}\right)}\left(1+|D v|^{p}\right) \mathrm{d} x .
$$

Hence, if $h>k$ we get

$$
\int_{\Omega}\left|D w_{h}-D w_{k}\right|^{p} \mathrm{~d} x=\sum_{i=k+1}^{h} \int_{G\left(x_{i}, r_{i}\right)}\left|D v_{i}-D v\right|^{p} \mathrm{~d} x \leq c \int_{\Omega^{\prime} \backslash\left(\cup_{i \leq k} G\left(x_{i}, r_{i}\right)\right)}\left(1+|D v|^{p}\right) \mathrm{d} x
$$

and the integral on the right hand side converges to zero as $k \rightarrow \infty$.

\subsection{The general case}

In the previous section we have proved Theorem 3.1 under the assumption that the bipolar function $f^{* *}$ coincides with the affine function $\langle m(\cdot), \xi\rangle+q(\cdot)$ on the set $A(x)=\left\{\xi \in \mathbb{R}^{n}: f(x, \xi)>f^{* *}(x, \xi)\right\}$ defined in (31). Here we consider the more general situation where the set $A(x)$ can be split into an union of a (at most countable) family of pairwise disjoint open sets $A_{j}(x)$, and that in each $A_{j}(x)$ the function $f^{* *}(x, \xi)$ coincides with an affine function $\left\langle m_{j}(x), \xi\right\rangle+q(x)$, where the slopes $m_{j}$ and the functions $q_{j}$ may vary with $j$.

Precisely, let $f: \Omega \times \mathbb{R}^{n} \rightarrow \mathbb{R}$ be a continuous function, satisfying the growth condition (28), the uniform convexity condition (29), and (30). As before, we assume that $f^{* *}$ is continuous and we denote by $\Omega_{A}$ the set of points $x \in \Omega$ such that $A(x) \neq \emptyset$. Moreover, we assume that, for all $x \in \Omega_{A}$, there exists a sequence $A_{j}(x)$ of pairwise disjoint open sets such that

$$
A(x)=\cup_{j} A_{j}(x),
$$

and that, for every $j$, the set $\Omega_{A_{j}}:=\left\{x \in \Omega: A_{j}(x) \neq \emptyset\right\}$ is open. Further, assume that, for all $j$, there exist a function $q_{j} \in C^{0}\left(\Omega_{A_{j}}\right)$ and a vector field $m_{j} \in C^{1}\left(\Omega_{A_{j}} ; \mathbb{R}^{n}\right)$ such that

$$
f^{* *}(x, \xi)=q_{j}(x)+\left\langle m_{j}(x), \xi\right\rangle, \quad \forall x \in \Omega_{A_{j}}, \xi \in A_{j}(x),
$$

and the boundary of the set where the divergence of $m_{j}$ is equal to zero is negligible; i.e., for all $j$,

$$
\operatorname{meas}\left(\Omega \cap \partial\left\{x \in \Omega_{A_{j}}: \operatorname{div} m_{j}(x)=0\right\}\right)=0 .
$$

For all $x \in \Omega_{A_{j}}$ set

$$
E_{j}(x):=\left\{\xi \in \mathbb{R}^{n}: f^{* *}(x, \xi)=q_{j}(x)+\left\langle m_{j}(x), \xi\right\rangle\right\}
$$

and we assume that, for every $j \in \mathbb{N}$, there exists an increasing function $\omega_{j}:[0,+\infty) \rightarrow[0,+\infty)$, with $\omega_{j}(t)=0$ if and only if $t=0$, such that, if $x \in \Omega_{A}, \xi \in \partial E_{j}(x), \eta \in \mathbb{R}^{n} \backslash E_{j}(x)$, then

$$
f^{* *}\left(x, \frac{\xi+\eta}{2}\right) \leq \frac{1}{2} f^{* *}(x, \xi)+\frac{1}{2} f^{* *}(x, \eta)-\omega_{j}(|\xi-\eta|) .
$$

As in the previous section we have the following existence result. 
Theorem 3.6. Let $f, f^{* *}: \Omega \times \mathbb{R}^{n} \rightarrow \mathbb{R}$ be continuous functions ( $f$ not necessarily convex with respect to $\left.\xi \in \mathbb{R}^{n}\right)$. Under the above assumptions on $f$ and $f^{* *}$, for any given boundary datum $u_{0} \in W^{1, p}(\Omega)$ the variational problem (27) attains its minimum. Moreover, any minimizers is of class $W_{\mathrm{loc}}^{1, \infty}(\Omega)$.

Remark 3.7. Notice that the assumptions (59) and (60) are equivalent to the following local assumption: for any $\left(x_{0}, \xi_{0}\right) \in \Omega \times \mathbb{R}^{n}$ such that $f\left(x_{0}, \xi_{0}\right)>f^{* *}\left(x_{0}, \xi_{0}\right)$ there exist $\delta>0$, a function $q \in C^{0}\left(B_{\delta}\left(x_{0}\right)\right)$, and a vector field $m \in C^{1}\left(B_{\delta}\left(x_{0}\right) ; \mathbb{R}^{n}\right)$ such that, if $\left|x-x_{0}\right|<\delta,\left|\xi-\xi_{0}\right|<\delta$, then

$$
f^{* *}(x, \xi)=q(x)+\langle m(x), \xi\rangle
$$

In fact, set $D:=\left\{(x, \xi) \in \Omega \times \mathbb{R}^{n}: f(x, \xi)>f^{* *}(x, \xi)\right\}$. The set $D$ is open, hence we may consider its connected components $D_{j}$. For every $x \in \Omega$ and every $j$ let us set $A_{j}(x):=\left\{\xi \in \mathbb{R}^{n}:(x, \xi) \in D_{j}\right\}$. For every $x$ the family $\left\{A_{j}(x)\right\}$ is a partition of $A(x):=\left\{\xi \in \mathbb{R}^{n}: f(x, \xi)>f^{* *}(x, \xi)\right\}$. Moreover, the set $\Omega_{A_{j}}:=\left\{x \in \Omega: A_{j}(x) \neq \emptyset\right\}$ is open, since it is equal to the projection of $D_{j}$ onto $\mathbb{R}^{n}$. Finally, the existence for every $j$ of a function $q_{j} \in C^{0}\left(\Omega_{A_{j}}\right)$ and a vector field $m_{j} \in C^{1}\left(\Omega_{A_{j}} ; \mathbb{R}^{n}\right)$ satisfying (60) is an immediate consequence of the local assumption (62) and of the connectedness of $D_{j}$.

To prove Theorem 3.6 one may follow exactly the same argument used in the proof of Theorem 3.1, with the obvious changes due to the fact that now we have to deal separately with the different affine representations (60) of $f^{* *}$. Therefore we shall limit ourselves to point out where the proof has to be modified.

The statement of Lemma 3.2 must be replaced by the following one.

Lemma 3.8. The minimum of the relaxed variational problem (35) is attained. Moreover, there exists a minimizer $v \in W_{\text {loc }}^{1, \infty}(\Omega) \cap\left(u_{0}+W^{1, p}(\Omega)\right)$ of (35) and there exist pairwise disjoint open sets $\Omega_{j}^{\prime} \subset \Omega$ (possibly empty) such that

$$
\left\{\begin{array}{lll}
D v(x) \in A_{j}(x) & \text { a.e. } & x \in \Omega_{j}^{\prime} \\
D v(x) \notin A(x) & \text { a.e. } & x \in \Omega \backslash \cup_{j} \Omega_{j}^{\prime}
\end{array}\right.
$$

and $\operatorname{div} m_{j}=0$ in $\Omega_{j}^{\prime}$.

Proof. To proof this lemma we argue as in the proof of Lemma 3.2, splitting each open set $\Omega_{A_{j}}$ into the three open sets

$$
\begin{gathered}
\Omega_{A_{j}}^{+}:=\left\{x \in \Omega_{A_{j}}: \operatorname{div} m_{j}(x)>0\right\}, \Omega_{A_{j}}^{-}:=\left\{x \in \Omega_{A_{j}}: \operatorname{div} m_{j}(x)<0\right\}, \\
\Omega_{A_{j}}^{0}:=\left\{x \in \Omega_{A_{j}}: \operatorname{div} m_{j}(x)=0\right\} .
\end{gathered}
$$

Taking a point $x_{0} \in \Omega_{A_{j}} \backslash \partial \Omega_{A_{j}}^{0}$ such that $u^{* *}$ is differentiable in $x_{0}$ and $D u^{* *}\left(x_{0}\right) \in A_{j}\left(x_{0}\right)$ for some $j$, we construct the function $v_{x_{0}}^{r}$ as before, noticing that the parameter $\gamma$ can be always chosen so small (see (46)) that

$$
D v_{x_{0}}^{r}(x) \in A_{j}(x) \quad \text { a.e. } x \in \bar{B}_{\delta}\left(x_{0}\right), \forall r \in(0, \delta]
$$

Arguing as in the model case, we get again a sequence $G\left(x_{k}, r_{k}\right)$ of pairwise disjoint sets such that the open set

$$
\Omega^{\prime}:=\bigcup_{\operatorname{int} G\left(x_{k}, r_{k}\right) \in \mathcal{G}^{\prime}} \operatorname{int} G\left(x_{k}, r_{k}\right)
$$

covers, up to a set of measure zero, the set of points $x_{0}$ where $u^{* *}$ is differentiable and $D u^{* *}\left(x_{0}\right) \in A_{j}\left(x_{0}\right)$ for some $j$. Therefore, if we define $v$ as in (52) we have again that $D v(x) \notin A(x)$ for a.e. $x \in \Omega \backslash \Omega^{\prime}$ and, by (63) 
that for any $k$ there exists $j_{k}$ such that

$$
D v(x) \in A_{j_{k}}(x) \quad \text { for a.e. } x \in G\left(x_{k}, r_{k}\right) \text {. }
$$

In particular $\Omega^{\prime}$ can be written as the disjoint union of the open sets

$$
\Omega_{j}^{\prime}=\bigcup\left\{\operatorname{int} G\left(x_{s}, r_{s}\right): D v_{x_{s}}^{r_{s}}(x) \in A_{j}(x) \quad \forall x \in G\left(x_{s}, r_{s}\right)\right\}
$$

and $D v(x) \in A_{j}(x)$ a.e. $x \in \Omega_{j}^{\prime}$, for all $j$. The rest of the proof follows with the obvious modifications.

Proof of Theorem 3.6. We claim that, for all $j$, the set function $x \in \Omega_{A_{j}} \mapsto E_{j}(x)$ is continuous in the sense of Kuratowski. It can be easily checked via the same argument used in Lemma 3.4, by virtue of assumption (61) (this is the only point where this hypothesis is needed). Therefore, in view of Lemma 3.5, for every $j$ the function $F_{j}$ defined by

$$
F_{j}(x, \xi):=\left\{\begin{array}{l}
\operatorname{dist}\left(\xi ; \partial E_{j}(x)\right) \text { if } \xi \in \mathbb{R}^{n} \backslash E_{j}(x) \\
-\operatorname{dist}\left(\xi ; \partial E_{j}(x)\right) \text { if } \xi \in E_{j}(x),
\end{array}\right.
$$

is continuous in $\Omega_{A_{j}} \times \mathbb{R}^{n}$ and convex with respect to $\xi$. Then, replacing (46) with (63), the proof goes on exactly as in the model case.

\section{Appendix: Some properties of the Distance Function}

In this section we prove some properties of the signed distance function to a convex set, which have been used in Section 3 in order to establish Lemma 3.4 and Lemma 3.5. We recall that if $E \subset \mathbb{R}^{n}$ then the signed distance function to $E$ is a function $d_{E}: \mathbb{R}^{n} \rightarrow \mathbb{R}$ defined as follows:

$$
d_{E}(\xi):= \begin{cases}\operatorname{dist}(\xi ; \partial E) & \text { if } \xi \notin E \\ -\operatorname{dist}(\xi ; \partial E) & \text { if } \xi \in E .\end{cases}
$$

Lemma 4.1. Let $E \subset \mathbb{R}^{n}$ be a closed convex set. If $\xi \in \mathbb{R}^{n}$ is such that

$$
\xi \in I_{\delta}(E), \quad \operatorname{dist}\left(\xi ; \partial I_{\delta}(E)\right)>\delta,
$$

for some $\delta>0$, then $\xi \in E$ (more precisely $\xi \in \operatorname{int} E$ ).

Proof. If $\xi \notin E$ then we consider the projection $\xi_{0}$ of $\xi$ on $E$ and a supporting hyperplane $H$ to $E$ through $\xi_{0}$. This hyperplane $H$ separates $\xi$ from $E$. Let us denote by $\eta$ the point

$$
\eta:=\xi+\delta \frac{\xi-\xi_{0}}{\left|\xi-\xi_{0}\right|}
$$

clearly $|\eta-\xi|=\delta$. Since by the assumptions $\bar{B}_{\delta}(\xi) \subset I_{\delta}(E)$, in particular $\eta \in I_{\delta}(E)$. On the other hand, $\operatorname{dist}(\eta ; E) \geq \operatorname{dist}(\eta ; H)=\left|\xi-\xi_{0}\right|+\delta>\delta$, which gives a contradiction.

Lemma 4.2. Let $E_{h}$ and $E$ be convex, compact sets of $\mathbb{R}^{n}$, such that $E_{h} \rightarrow E$ in the sense of Kuratowski. Then

$$
d(\xi)=\lim _{h \rightarrow+\infty} d_{h}(\xi), \quad \forall \xi \in \mathbb{R}^{n},
$$

where $d_{h}$ and $d$ are, respectively, the signed distance functions to $E_{h}$ and $E$. 
Proof. Note that we use the assumption of convexity of the sets $E_{h}, E$ only in Steps 2 and 3 below.

Step 1: Let us consider the case where $\xi \notin E$. Then there exists $\varepsilon>0$ such that dist $(\xi ; \partial E)>\varepsilon$ and, for large $h \in \mathbb{N}, \xi \notin E_{h}$. Let $\xi_{0} \in E$ be a point such that $d(\xi)=\left|\xi-\xi_{0}\right|$, and let $\xi_{h}$ be a sequence of points in $E_{h}$ converging to $\xi_{0}$. Then

$$
d(\xi)=\lim _{h \rightarrow+\infty}\left|\xi-\xi_{h}\right| \geq \limsup _{h \rightarrow+\infty} d_{h}(\xi)
$$

Conversely, let $\xi_{h_{k}} \in E_{h_{k}}$ be such that

$$
\liminf _{h \rightarrow+\infty} d_{h}(\xi)=\lim _{k \rightarrow+\infty}\left|\xi-\xi_{h_{k}}\right|
$$

By extracting, if necessary, a further subsequence, we may assume that $\xi_{h_{k}} \rightarrow \xi_{0}$, for some $\xi_{0} \in E$. Thus

$$
\liminf _{h \rightarrow+\infty} d_{h}(\xi)=\lim _{k \rightarrow+\infty}\left|\xi-\xi_{h_{k}}\right|=\left|\xi-\xi_{0}\right| \geq d(\xi) .
$$

Step 2: Let $\xi \in \partial E$. There exists a sequence $h_{k}$ such that

$$
\limsup _{h \rightarrow+\infty} d_{h}(\xi)=\lim _{k \rightarrow+\infty} d_{h_{k}}(\xi) .
$$

Let $\xi_{h_{k}} \in E_{h_{k}}$ be such that $\xi_{h_{k}} \rightarrow \xi$. Since $\left|\xi-\xi_{h_{k}}\right| \geq d_{h_{k}}(\xi)$, we obtain

$$
\limsup _{h \rightarrow+\infty} d_{h}(\xi) \leq 0=d(\xi)
$$

Suppose now that $\liminf _{h \rightarrow+\infty} d_{h}(\xi)<0$. As before, there exists a sequence $h_{k}$ such that $\liminf _{h \rightarrow+\infty} d_{h}(\xi)=$ $\lim _{k \rightarrow+\infty} d_{h_{k}}(\xi)$. Fix $\varepsilon>0$. For $k$ large enough $d_{h_{k}}(\xi)<0$ and $\xi \in E_{h_{k}} \subset I_{\varepsilon}(E)$. Let us denote by $\nu$ a unit vector in $\mathbb{R}^{n}$, orthogonal to a supporting hyperplane to $E$ at $\xi$, pointing to the exterior of $E$. By the convexity of $E$, the vector $\xi+2 \varepsilon \nu \notin I_{\varepsilon}(E)$, and so $\xi+2 \varepsilon \nu \notin E_{h_{k}}$ for large $k$. Therefore, for such $k$, $\operatorname{dist}\left(\xi ; \partial E_{h_{k}}\right)=\left|d_{h_{k}}(\xi)\right|<2 \varepsilon$. This implies that $\liminf _{h \rightarrow+\infty} d_{h}(\xi) \geq 0$.

Step 3: Let $\xi \in \operatorname{int} E$ and fix $\delta \in(0$, dist $(\xi ; \partial E))$ such that $B_{\delta}(\xi) \subset E$. For every $\varepsilon<\delta$ there exists $h_{\varepsilon}$ such that $E \subset I_{\varepsilon}\left(E_{h}\right)$ for $h>h_{\varepsilon}$. If $\eta \in B_{\delta-\varepsilon}(\xi) \subset E \subset I_{\varepsilon}\left(E_{h}\right)$, then $\operatorname{dist}\left(\eta ; \partial I_{\varepsilon}\left(E_{h}\right)\right)>\varepsilon$; therefore, by Lemma 4.1, we have that $B_{\delta-\varepsilon}(\xi) \subset E_{h}$ for $h>h_{\varepsilon}$ (in particular $\left.\xi \in \operatorname{int} E_{h}\right)$ and dist $\left(\xi ; \partial E_{h}\right) \geq \delta-\varepsilon$. From this it follows that $\liminf _{h \rightarrow+\infty} \operatorname{dist}\left(\xi ; \partial E_{h}\right)=\liminf _{h \rightarrow+\infty}\left[-d_{h}(\xi)\right] \geq \delta$. Letting $\delta \rightarrow \operatorname{dist}(\xi ; \partial E)$, we get

$$
d(\xi) \geq \limsup _{h \rightarrow+\infty} d_{h}(\xi)
$$

Conversely, let $\xi_{0} \in \partial E$ be such that $\left|\xi-\xi_{0}\right|=\operatorname{dist}(\xi ; \partial E)$. From Step 2 it follows that there exists $\xi_{h} \in \partial E_{h}$ such that $\left|\xi_{h}-\xi_{0}\right| \rightarrow 0$. We also have

$$
\operatorname{dist}\left(\xi ; \partial E_{h}\right) \leq\left|\xi_{h}-\xi\right| \leq\left|\xi_{h}-\xi_{0}\right|+\operatorname{dist}(\xi ; \partial E)
$$

Since $\xi \in E_{h}$,

$$
\liminf _{h \rightarrow+\infty} d_{h}(\xi)=\liminf _{h \rightarrow+\infty}\left[-\operatorname{dist}\left(\xi ; \partial E_{h}\right)\right] \geq-\operatorname{dist}(\xi ; \partial E)=d(\xi)
$$

and the proof is complete. 
In the next lemma we prove the convexity of the signed distance function to a convex set.

Lemma 4.3. If $E$ is a compact, convex subset of $\mathbb{R}^{n}$ then the signed distance $d_{E}$, defined in (64), is a convex function.

Proof. The signed distance from an half space is an affine function. Therefore the signed distance $d_{E}$ is convex, since it is the supremum of the family of signed distance functions from all the half spaces containing $E$.

The research of Irene Fonseca was partially supported by the National Science Foundation under Grant No. DMS9731957. The research of Nicola Fusco and Paolo Marcellini was partially supported by the Italian Ministero dell'Università e della Ricerca Scientifica e Tecnologica (MURST). The authors acknowledge the support of the Center for Nonlinear Analysis under NSF Grant No. DMS-9803791.

\section{REFERENCES}

[1] E. Acerbi and N. Fusco, Regularity for minimizers of nonquadratic functionals: The case $1<p<2$. J. Math. Anal. Appl. 140 (1989) 115-135.

[2] L. Ambrosio, N. Fusco and D. Pallara, Special functions of bounded variation and free discontinuity problems. Oxford University Press (2000).

[3] J.M. Ball and R.D. James, Fine phase mixtures as minimizers of energy. Arch. Rational Mech. Anal. 100 (1987) 15-52.

[4] J.M. Ball and R.D. James, Proposed experimental tests of a theory of fine microstructure and the two wells problem. Philos. Trans. Roy. Soc. London Ser. A 338 (1991) 389-450.

[5] P. Celada and S. Perrotta, Minimizing non convex, multiple integrals: A density result. Proc. Roy. Soc. Edinburgh Sect. A 130 (2000) 721-741.

[6] A. Cellina, On minima of a functional of the gradient: Necessary conditions. Nonlinear Anal. 20 (1993) 337-341.

[7] A. Cellina, On minima of a functional of the gradient: Sufficient conditions. Nonlinear Anal. 20 (1993) 343-347.

[8] B. Dacorogna and P. Marcellini, Existence of minimizers for non quasiconvex integrals. Arch. Rational Mech. Anal. 131 (1995) 359-399.

[9] B. Dacorogna and P. Marcellini, Théorème d'existence dans le cas scalaire et vectoriel pour les équations de Hamilton-Jacobi. C. R. Acad. Sci. Paris Sér. I Math. 322 (1996) 237-240.

[10] B. Dacorogna and P. Marcellini, Sur le problème de Cauchy-Dirichlet pour les systèmes d'équations non linéaires du premier ordre. C. R. Acad. Sci. Paris Sér. I Math. 323 (1996) 599-602.

[11] B. Dacorogna and P. Marcellini, General existence theorems for Hamilton-Jacobi equations in the scalar and vectorial case. Acta Math. 178 (1997) 1-37.

[12] B. Dacorogna and P. Marcellini, Implicit partial differential equations. Birkhäuser, Boston (1999).

[13] B. Dacorogna and P. Marcellini, Attainment of minima and implicit partial differential equations. Ricerche Mat. 48 (1999) 311-346.

[14] F.S. De Blasi and G. Pianigiani, On the Dirichlet problem for first order partial differential equations. A Baire category approach. NoDEA Nonlinear Differential Equations Appl. 6 (1999) 13-34.

[15] G. Dolzmann, B. Kirchheim, S. Müller and V. Šverák, The two-well problem in three dimensions. Calc. Var. Partial Differential Equations 10 (2000) 21-40.

[16] L.C. Evans, Quasiconvexity and partial regularity in the calculus of variations. Arch. Rational Mech. Anal. 95 (1986) $227-252$.

[17] L.C. Evans and R.F. Gariepy, Blowup, compactness and partial regularity in the calculus of variations. Indiana Univ. Math. J. 36 (1987) 361-371.

[18] I. Fonseca and G. Francfort, $3 D-2 D$ asymptotic analysis of an optimal design problem for thin films. J. Reine Angew. Math. 505 (1998) 173-202.

[19] I. Fonseca and N. Fusco, Regularity results for anisotropic image segmentation models. Ann. Scuola Norm. Sup. Pisa Cl. Sci. (4) 24 (1997) 463-499.

[20] I. Fonseca and G. Leoni, Bulk and contact energies: Nucleation and relaxation. SIAM J. Math. Anal. 30 (1998) 190-219.

[21] G. Friesecke, A necessary and sufficient condition for non attainment and formation of microstructure almost everywhere in scalar variational problems. Proc. Royal Soc. Edinburgh Sect. A 124 (1994) 437-471. 
[22] P. Marcellini, A relation between existence of minima for nonconvex integrals and uniqueness for not strictly convex integrals of the calculus of variations, Math. Theories of Optimization, edited by J.P. Cecconi and T. Zolezzi. Springer-Verlag, Lecture Notes in Math. 979 (1983) 216-231.

[23] E. Mascolo and R. Schianchi, Existence theorems for nonconvex problems. J. Math. Pures Appl. 62 (1983) $349-359$.

[24] E. Mascolo and R. Schianchi, Nonconvex problems in the calculus of variations. Nonlinear Anal. 9 (1985) 371-379.

[25] E. Mascolo and R. Schianchi, Existence theorems in the calculus of variations. J. Differential Equations 67 (1987) $185-198$.

[26] S. Müller and V. Šverák, Attainment results for the two-well problem by convex integration, edited by J. Jost. International Press (1996) 239-251.

[27] J.P. Raymond, Existence of minimizers for vector problems without quasiconvexity conditions. Nonlinear Anal. 18 (1992) $815-828$.

[28] M.A. Sychev, Characterization of homogeneous scalar variational problems solvable for all boundary data. Proc. Roy. Soc. Edinburgh Sect. A 130 (2000) 611-631.

[29] S. Zagatti, Minimization of functionals of the gradient by Baire's theorem. SIAM J. Control Optim. 38 (2000) $384-399$.

[30] W.P. Ziemer, Weakly differentiable functions. Springer-Verlag, New York, Grad. Texts in Math. (1989). 